\title{
Emotions in Team Contact Sports: A Systematic Review
}

\author{
Mickaël Campo \\ Université Lyon \\ Stephen Mellalieu \\ Swansea University \\ Claude Ferrand \\ Université François Rabelais \\ Guillaume Martinent \\ Université Lyon \\ Elisabeth Rosnet
}

Institut National du Sport de l'Expertise et de la Performance

This study systematically reviewed the literature on the emotional processes associated with performance in team contact sports. To consider the entire emotional spectrum, Lazarus's (1999) cognitive motivational relational theory was used as a guiding framework. An electronic search of the literature identified 48 of 5,079 papers as relevant. Anxiety and anger were found to be the most common emotions studied, potentially due to the combative nature of team contact sports. The influence of group processes on emotional experiences was also prominent. The findings highlight the need to increase awareness of the emotional experience in team contact sports and to develop emotion-specific regulation strategies. Recommendations for future research include exploring other emotions that might emerge from situations related to collisions (e.g., fright) and emotions related to relationships with teammates (e.g., guilt and compassion).

Numerous investigations have highlighted that emotion is an inherent part of the competitive experience and influences performance (Hanin, 2000; Jones, 2003; Lazarus, 2000). However, many terms (e.g., as mood and affect) are used in the

Campo and Martinent are with the Centre de Recherche et d'Innovation sur le Sport, Universite Lyon, Villeurbanne, France. Ferrand is with the Laboratoire de Psychologie des Âges de la Vie, Université FranÇois Rabelais, Tours, France. Mellalieu is with the Dept. of Sports Science, Swansea University, Swansea, UK. Rosnet is with Institut National du Sport de l'Expertise et de la Performance-Mission Recherche, Paris, France. 

study of emotion, such that defining what an emotion is can be difficult. Affect refers to the experiential component of all valenced responses (Lane, Beedie, \& Stevens, 2005). They include constructs such as emotions and moods (Frijda, 1994; Oatley \&Jenkins, 1996). Emotions are directly related to specific stimuli, more intense and of a shorter duration compared with moods (Parkinson, Totterdell, Briner, \& Reynolds, 1996). For example, contentedness or fatigue are related to global affective states based on daily experience and are recognized as moods. Conversely, emotions such as happiness or sadness are not only more intense, but also discrete reactions to specific events (Biddle, 2000).

The role of cognition in emotion has long been acknowledged in sport psychology (e.g., Cerin, Szabo, Hunt, \&Williams, 2000). Lazarus's (1999) cognitive motivational relational theory (CMRT) advocates a contemporary approach to the cognitive process involved in generating specific emotions (Jones, 2003). Emerging from the transactional approach to the stress process (Lazarus \& Folkman, 1984), CMRT provides a framework to study emotions in sports (Lazarus, 2000; Uphill \& Jones, 2007). According to Lazarus, emotions are the personal expression of social life events that arise when a person appraises a situation as relevant to his current goals (Lazarus, 1999). Lazarus posited that an emotion originates from a complex interaction between a person and his environment. Specifically, the cognitive appraisal of this transaction creates the emotions recognized in CMRT (i.e., anger, anxiety, fright, guilt, shame, sadness, envy, jealousy, disgust, happiness, pride, relief, hope, love, gratitude, and compassion; Lazarus, 1991; 1999; 2000). Consequently, each emotion is defined by its core relational theme, which is a summary of the primary and the second appraisals, with the first and the second appraisal relating to personal goals and coping options respectively. To illustrate how CMRT can be applied to sport, consider a rugby player tackling an opponent. The stimulus (tackling the opponent) is likely to be appraised as threatening and might cause anxiety (i.e., "facing uncertain, existential threat"; Lazarus, 1999, p. 96). Conversely, a player with a painful shoulder might appraise the same event as "an immediate, concrete, and overwhelming physical danger" - thus causing fright.

Emotional experiences may be influenced by both individual and situational variables (Lazarus, 1999; Ruiz \& Hanin, 2004; see Figure 1). The characteristics and requirements of the sport (i.e., sport type) moderate emotional experiences (Cerin etal., 2000; Dunn\&Nielsen, 1996; Martens, Vealey,\&Burton, 1990). Many ofthe inconsistent results regarding the relationship between emotion and performance may therefore be due to a comparison of results from different sport types. The sport psychology literature uses many classifications of sports (cf. Famose, 1990). For example, Vom Hofe (1982) classified sports by their social interactions (i.e., individual vs. team sports), environments (i.e., natural vs. standardized environments), and the emotions connected to their physicality (i.e., contact vs. noncontact sports). These objective characteristics intrinsicto specific sports havesubsequently been shown to influence anxiety and other sports-related emotions (Martens et al., 1990). Other researchers, such as Oxendine (1970), have classified sports based on the activation associated with the task required, whereas Dunn and Nielsen (1996) classified team sports based on the situational factors that lead to stress experiences.

Given that each sport has its own psychological characteristics (Cerin et al., 2000; Prapavessis \& Grove, 1994; Terry, 1995), examining sports according to their specific requirements is needed to understand the specific emotional experiences 


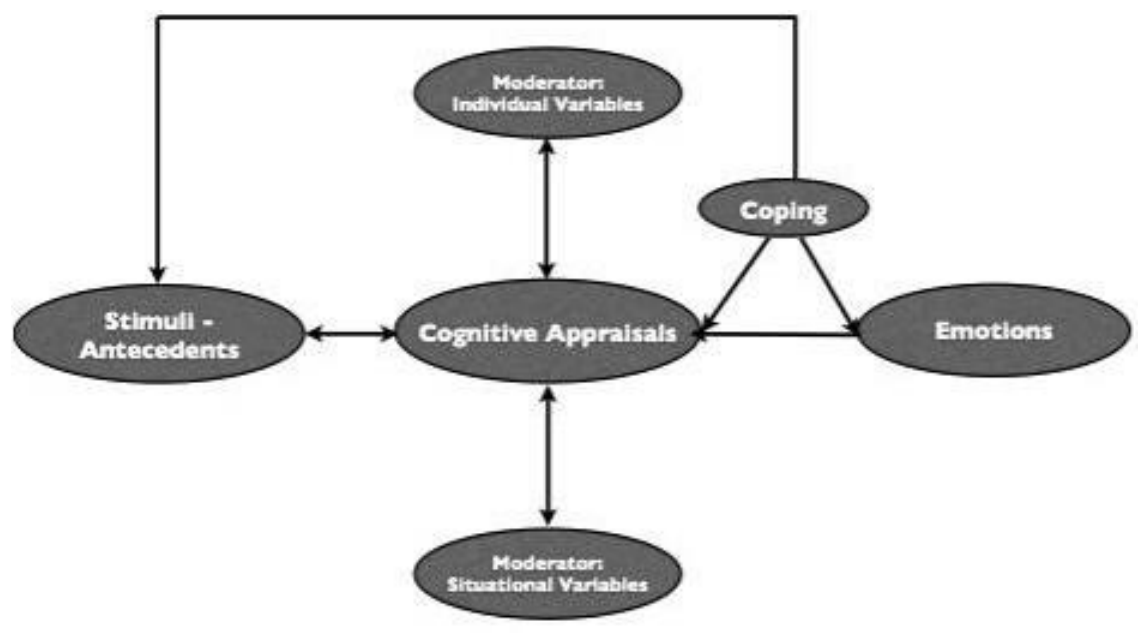

Figure 1 - Individual and situational variables in emotional experiences.

surrounding performance (Cerin et al., 2000). Until now, the literature has treated the individual-versus team-based sports dichotomy as merely performing alone or with others (Hanin, 2000). In relation to the study of emotion, one of interests of this dichotomy is that teammates can influence another player's emotional state (Totterdell, 2000). For example, one player's mistake may place another player in a situation where he could fail. Indeed, interactions with others can reduce a player's control of a situation (Mann, Williams, Ward, \&Janelle, 2007). Conversely, teammates offer a way to cope with one's emotions (Niven, Totterdell, \& Holman, 2009). However, integrating the degree of physicality into the categorization of team sports also seems pertinent (Isberg, 2000), specifically the notion of contact versus collision sports. Contact team sports, such as football or basketball, are distinctly different from collision sports (e.g., ice hockey and rugby league), not only in their rules, but also in the potential emotional experiences elicited through the direct and intense physicality involved in these sports (Maxwell, Visek, \& Moores, 2009).

Based upon Martens et al.'s (1990) differentiation between individual (e.g., skiing) versus team sports (e.g., volleyball), and contact (e.g., boxing) versus noncontact sports (e.g., dance and basketball), we chose to study team contact sports, also labeled as collective combative games (Conquet \& Devaluez, 1978), or as collision sports (Maxwell et al., 2009; Robazza, Bertollo, \& Bortoli, 2006). These sports group the notion of team and the notion of contact. Here, the rules legitimize acts of aggression (Lauer \& Paiement, 2009). Specifically, we were interested in the six major team contact sports (i.e., rugby union, rugby league, Australian rules football, Canadian rules football, U.S. football and ice hockey). Due to their intense physicality, researchers have acknowledged the particularly emotional nature of team contact sports (e.g., Lauer \& Paiement, 2009; Maxwell et al., 2009). They represent a rich source of potential knowledge to understand the emotional experience related to performance. Consequently, the aim of this study was to review the literature on the emotional processes associated with performance 
in team contact sports using Lazarus's CMRT (1999) as a framework to suggest specific directions for future research and applied practice.

\section{Method}

\section{Study Design}

For the purpose of this study, we performed a systematic review of the literature. Although meta-analyses are commonly used, this technique was not appropriate in our case because meta-analyses are only applicable when data are homogenous across studies (Eysenk, 1995). In addition, meta-analysis only yields "similar quantitative outcomes" (Bland, Meurer, \& Maldonado, 1995). Consequently, when the data, sample sizes, and variables are heterogeneous in nature, nonstatistical synthesis is preferred (Eysenk, 1995). The literature on team contact sports has a high level of heterogeneity in terms of study design, theoretical models, variables,

and so forth. Consequently, the variables studied, as well as the instruments used to collect them vary significantly across studies. Moreover, not every emotional process variable was considered across the numerous studies potentially available for review. Thus, as a quantitative meta-analytic literature review was not suitable for our research question, we adopted a nonstatistical synthesis, also known as a systematic review (Bland et al., 1995).

A systematic review is a scientific exercise for describing the current state of knowledge in a specific field to provide recommendations for future research and practical interventions (Murlow, 1995). To comprehensively represent the literature investigating the emotions associated with performance in team contact sports, we followed Weed's methodological guidelines for reviews (Weed, 1997). Specifically, we conducted a five-step procedure to protect the data from bias, and to inform the reader about our methods (state purpose, search, evaluate quality, summarize evidence, and conclude).

\section{Procedure}

In relation to the emotions associated with performance in team contact sports, we sought to provide directions for future research and to derive applied practice implications. To achieve these objectives, we conducted an exhaustive search of the literature to locate all the published studies in relation to the emotions associated with competing in team contact sports. We performed an electronic search using several online databases including PsycARTICLES, PsycINFO, PubMed, and SPORTDiscus. The first keywords used included emotion, stress, mood, affect, rugby, Canadian football, Australian football, US football, football and ice hockey. Wealso included all the emotions recognized by Lazarus (2000) as occurring in competitive sport (i.e., anger, anxiety, shame, pride, relief, happiness, and guilt). We limited our period from January 1985 to January 2010 to minimize conclusion biases based on older results irrelevant to contemporary team contact sports. For example, recent innovations in protective clothing, rule changes, and the evolution of a sport toward more intense and frequent collisions have significantly changed the way contact team sports are played compared with 20 years ago (Krauss, 2004). PsycARTICLES, PsycINFO, PubMed, and SPORTDiscus yielded 1,231, $2,464,914$, and 470 citations, respectively. After selecting only relevant articles, we entered these data into an Excel database. 
We considered only participants from studies of team contact sports for review. We excluded other team sports (e.g., soccer) or specific populations (e.g., match officials, coaches) from this study. Finally, we also excluded articles not directly related to emotion and performance such as those dealing with performer health (e.g., burnout or injury).

To account for the potential variations in the quality of the literature, only fulllength peer-reviewed scientific journal articles were initially selected for review. In addition, because peer-reviewed articles themselves vary in terms of quality, we used an adaptation of Bland et al.'s (1995) procedure for rating journal articles. Rating the quality of a study protects the data from bias and allowed us to include only papers of sufficient quality. Specifically, we rated the quality of each study by assessing its study design, data source(s), and level of theory determined by the presence of a theory description serving as the basis and not merely as an explanation of the results (see Bland et al., 1995, p. 647-648 for details regarding scoring anchors). While Bland et al. (1995) also rated the sample sizes of papers we chose not to use this index as we included qualitative studies, which would have made sample size comparisons difficult and potentially meaningless. To score each variable, Bland et al. (1995) used a range of assessment scales (i.e., sample sizes [0-20 points], study design [0-40 points], data source [0-25 points], and level of theory [0-15 points]). For the purposes of our review, however, we considered each of the three variables used as equally important ( $0-5$ points per scale). Consequently, a maximum of 15 points was possible. Bland et al. (1995) deemed that a paper scoring 45 points out of a possible 100 was of sufficient quality to include in a review. We adhered to this criterion by applying a $45 \%$ threshold to each variable whereby papers scoring at least 6.75 out of 15 were included in this review. The rating procedure was conducted by the first and second authors of this paper, of whom the former was also an expert in rugby union with 24 years of combined coaching and playing experience. In line with Bland et al.'s recommendations, we summarized information regarding the reviewed studies in a table (Table 1) to allow "readers to judge the proper weight to give the findings of the synthesis" (Bland et al., 1995; p. 643).

In the final stage of the procedure, the first and second author reviewed the remaining 48 papers and analyzed them in relationship to their respective results and methods. These findings were then categorized according to the specific elements of Lazarus's (1999) CMRT: (a) antecedents of emotions; (b) moderators of emotional states; (c) emotions experienced; and (d) subsequent coping responses.

\section{Results}

Our initial search generated 5,079 results of which 48 studies published between January 1985 and January 2010 were reviewed. Table 1 provides a detailed overview of this search, and Table 2 summarizes the results in relation to the elements of Lazarus's (1999) CMRT.

\section{Antecedents of Emotions}

Twelve papers investigated the antecedents of emotions in team contact sports by providing an overview of the major and specific environmental demands (i.e., stressors) from which players' emotional experiences originate. Twenty-one 
Table 1 Characteristics of Research Studying Emotions in Contact Team-Based Sports

\begin{tabular}{|c|c|c|c|c|c|c|c|c|c|c|c|}
\hline \multirow[b]{2}{*}{ Authors } & \multirow[b]{2}{*}{ Year } & \multirow{2}{*}{$\begin{array}{l}\text { Sample } \\
\text { size }\end{array}$} & \multicolumn{4}{|c|}{ Sample characteristics } & \multirow[b]{2}{*}{ Study question } & \multirow{2}{*}{$\begin{array}{l}\text { Study } \\
\text { design }\end{array}$} & \multirow{2}{*}{$\begin{array}{l}\text { Basic theories } \\
\text { of emotions }\end{array}$} & \multirow[b]{2}{*}{ Measures } & \multirow{2}{*}{$\begin{array}{l}\text { Rated } \\
\text { Qual. }\end{array}$} \\
\hline & & & Age & Level & Culture & Sport & & & & & \\
\hline \multirow{2}{*}{$\begin{array}{l}\text { (1) Grange \& } \\
\text { Kerr }\end{array}$} & \multirow[t]{2}{*}{2010} & $N=8$ & Und. & \multirow[t]{2}{*}{ Elite } & \multirow[t]{2}{*}{ Australian } & \multirow{2}{*}{$\begin{array}{l}\text { Australian } \\
\text { football }\end{array}$} & \multirow{2}{*}{$\begin{array}{l}\text { Examination of } \\
\text { different types of } \\
\text { aggressions }\end{array}$} & \multirow[t]{2}{*}{ Descriptive } & \multirow[t]{2}{*}{ Reversal theory } & \multirow[t]{2}{*}{ Interv. } & \multirow[t]{2}{*}{12} \\
\hline & & $\mathrm{n}_{\mathrm{s}}=8$ & & & & & & & & & \\
\hline \multirow{3}{*}{$\begin{array}{l}\text { (2) Gaudreau, } \\
\text { Amiot \& } \\
\text { Vallerand }\end{array}$} & \multirow[t]{3}{*}{2009} & \multirow{3}{*}{$\begin{array}{l}N=265 \\
\mathrm{n}_{\mathrm{s}}=265\end{array}$} & $M_{\text {age }}=$ & \multirow[t]{3}{*}{ Elite } & \multirow[t]{3}{*}{ Canadian } & \multirow{3}{*}{$\begin{array}{l}\text { Ice } \\
\text { hockey }\end{array}$} & \multirow{3}{*}{$\begin{array}{l}\text { Examination of } \\
\text { affective trajec- } \\
\text { tories }\end{array}$} & \multirow{3}{*}{$\begin{array}{l}\text { Preexperi- } \\
\text { mental }\end{array}$} & \multirow{3}{*}{$\begin{array}{l}\text { Positive- } \\
\text { Negative affect } \\
\text { approach }\end{array}$} & Quest. \& & \multirow[t]{3}{*}{18} \\
\hline & & & 25.20 & & & & & & & Scales & \\
\hline & & & $S D=$ Und & & & & & & & & \\
\hline \multirow{3}{*}{$\begin{array}{l}\text { (3) Géczi, Tóth, } \\
\text { Sipos, Fügedi, } \\
\text { Dancs \& } \\
\text { Bognár }\end{array}$} & \multirow[t]{3}{*}{2009} & $N=95$ & Ages & \multirow[t]{3}{*}{ Elite } & \multirow[t]{3}{*}{ Hungarian } & \multirow{3}{*}{$\begin{array}{l}\text { Ice } \\
\text { hockey }\end{array}$} & \multirow{3}{*}{$\begin{array}{l}\text { Examination } \\
\text { of psychologi- } \\
\text { cal determinants } \\
\text { related to perfor- } \\
\text { mance }\end{array}$} & \multirow{3}{*}{$\begin{array}{l}\text { Observa- } \\
\text { tional }\end{array}$} & Cognitive- & Quest. & 13 \\
\hline & & $\mathrm{n}_{\mathrm{s}}=95$ & $\begin{array}{l}\text { ranged } \\
\text { from } 15 \text { to }\end{array}$ & & & & & & $\begin{array}{l}\text { Somatic anxiety } \\
\text { approach }\end{array}$ & & \\
\hline & & & $19 \mathrm{yrs}$. & & & & & & & & \\
\hline (4) Lauer \& & 2009 & $N=3$ & Ages & Non & Und. & Ice & Examination of a & Descriptive & Lazarus-CMR & Obs. \& & 8 \\
\hline Paiement & & $\mathrm{n}_{\mathrm{s}}=3$ & $\begin{array}{l}\text { ranged } \\
\text { from } 12 \text { to }\end{array}$ & elite & & hockey & $\begin{array}{l}\text { program effective- } \\
\text { nessused to reduce }\end{array}$ & & & Interv. & \\
\hline & & & $14 \mathrm{yrs}$ & & & & $\begin{array}{l}\text { aggressive behav- } \\
\text { iors }\end{array}$ & & & & \\
\hline (5) Maxwell \& & 2009 & $N=144$ & $M_{\text {age }}=$ & Elite & Chinese & Rugby & Examination of & Observa- & Aggressiveness & Quest. & 8 \\
\hline Vis & & $n_{s}=144$ & $\begin{array}{l}\text { Und. } \\
S D=\text { Und. }\end{array}$ & $\begin{array}{l}\& \text { non } \\
\text { elite }\end{array}$ & & u & $\begin{array}{l}\text { the relationships } \\
\text { between aggres- } \\
\text { siveness, anger, } \\
\text { athletic identityand } \\
\text { professionalization }\end{array}$ & & & & \\
\hline
\end{tabular}


Table 1 (continued)

\begin{tabular}{|c|c|c|c|c|c|c|c|c|c|c|c|}
\hline \multirow[b]{2}{*}{ Authors } & \multirow[b]{2}{*}{ Year } & \multirow{2}{*}{$\begin{array}{l}\text { Sample } \\
\text { size }\end{array}$} & \multicolumn{4}{|c|}{ Sample characteristics } & \multirow[b]{2}{*}{ Study question } & \multirow{2}{*}{$\begin{array}{l}\text { Study } \\
\text { design }\end{array}$} & \multirow{2}{*}{$\begin{array}{c}\text { Basic theories } \\
\text { of emotions }\end{array}$} & \multirow[b]{2}{*}{ Measures } & \multirow{2}{*}{$\begin{array}{l}\text { Rated } \\
\text { Qual. }\end{array}$} \\
\hline & & & Age & Level & Culture & Sport & & & & & \\
\hline \multirow{4}{*}{$\begin{array}{l}\text { (6) Maxwell, } \\
\text { Visek \& Moores }\end{array}$} & \multirow{4}{*}{2009} & \multirow{4}{*}{$\begin{array}{l}N=471 \\
\mathrm{n}_{\mathrm{s}}=82\end{array}$} & $M_{\text {age }}=$ & \multirow[t]{4}{*}{ Und. } & \multirow[t]{4}{*}{ Chinese } & \multirow{4}{*}{$\begin{array}{l}\text { Rugby } \\
\text { union }\end{array}$} & \multirow{4}{*}{$\begin{array}{l}\text { Examination of } \\
\text { the relationship } \\
\text { between aggres- } \\
\text { siveness and anger } \\
\text { ineastern culture }\end{array}$} & \multirow{4}{*}{$\begin{array}{l}\text { Observa- } \\
\text { tional }\end{array}$} & \multirow{4}{*}{$\begin{array}{l}\text { Cultural } \\
\text { approach }\end{array}$} & \multirow[t]{4}{*}{ Quest. } & \multirow[t]{4}{*}{11} \\
\hline & & & 25.20 & & & & & & & & \\
\hline & & & $S D=8.94$ & & & & & & & & \\
\hline & & & & & & & & & & & \\
\hline \multirow{6}{*}{$\begin{array}{l}\text { (7) Mellalieu, } \\
\text { Hanton \& } \\
\text { Thomas }\end{array}$} & \multirow{6}{*}{2009} & \multirow{6}{*}{$\begin{array}{l}N=5 \\
\mathrm{n}_{\mathrm{s}}=5\end{array}$} & $M_{\text {age }}=$ & \multirow{6}{*}{$\begin{array}{l}\text { Non } \\
\text { elite }\end{array}$} & \multirow[t]{6}{*}{ Und. } & \multirow{6}{*}{$\begin{array}{l}\text { Rugby } \\
\text { union }\end{array}$} & \multirow{6}{*}{$\begin{array}{l}\text { Examination of } \\
\text { effects of moti- } \\
\text { vational general- } \\
\text { arousal imagery- } \\
\text { intervention upon } \\
\text { preperformance } \\
\text { symptoms }\end{array}$} & \multirow{6}{*}{$\begin{array}{l}\text { Preexperi- } \\
\text { mental }\end{array}$} & \multirow{6}{*}{$\begin{array}{l}\text { Directional } \\
\text { approach }\end{array}$} & \multirow[t]{6}{*}{ Quest. } & \multirow[t]{6}{*}{16} \\
\hline & & & 24.05 & & & & & & & & \\
\hline & & & $S D=3.05$ & & & & & & & & \\
\hline & & & & & & & & & & & \\
\hline & & & & & & & & & & & \\
\hline & & & & & & & & & & & \\
\hline \multirow{2}{*}{$\begin{array}{l}\text { (8) Nich- } \\
\text { olls, Jones, } \\
\text { Polman \& } \\
\text { Borkoles }\end{array}$} & \multirow[t]{2}{*}{2009} & $N=5$ & $M_{\mathrm{age}}=27.2$ & Elite & British & Rugby & Examination of & Descriptive & Lazarus-CMR & Daily- diaries & 8 \\
\hline & & $\mathrm{n}_{\mathrm{s}}=5$ & $S D=5.7$ & & & union & $\begin{array}{l}\text { stressors, coping } \\
\text { and emotions }\end{array}$ & & & sheets & \\
\hline (9) Nicholls, & 2009 & $N=16$ & $M_{\mathrm{age}}=19.3$ & Elite & English & Rugby & Examination of & Descriptive & Lazarus-CMR & Quest. & 10 \\
\hline $\begin{array}{l}\text { Backhouse, } \\
\text { Polman \& } \\
\text { McKenna }\end{array}$ & & $\mathrm{n}_{\mathrm{s}}=16$ & $S D=0.95$ & & & union & $\begin{array}{l}\text { affects and sources } \\
\text { of stress in differ- } \\
\text { ent periods (train- } \\
\text { ing, rest, match) }\end{array}$ & & & & \\
\hline (10) Géczi, & 2008 & $N=52$ & $M_{\text {age young }}=$ & Elite & Hungariar & Ice & Examination of & Observa- & Cognitive- & Quest. & 13 \\
\hline $\begin{array}{l}\text { Bognár, Tóth, } \\
\text { Sipos\& }\end{array}$ & & $\mathrm{n}_{\mathrm{s}}=52$ & 16.78 & & & Hockey & psychological & tional & Somatic anxiety & & \\
\hline Fügedi & & & $\begin{array}{l}M_{\text {age adult }}= \\
27.21\end{array}$ & & & & ence performance & & & & \\
\hline & & & $S D=U n d$ & & & & $\begin{array}{l}\text { related to the age of } \\
\text { the participants }\end{array}$ & & & & \\
\hline
\end{tabular}




\begin{tabular}{|c|c|c|c|c|c|c|c|c|c|c|c|}
\hline \multirow[b]{2}{*}{ Authors } & \multirow[b]{2}{*}{ Year } & \multirow{2}{*}{$\begin{array}{l}\text { Sample } \\
\text { size }\end{array}$} & \multicolumn{4}{|c|}{ Sample characteristics } & \multirow[b]{2}{*}{ Study question } & \multirow{2}{*}{$\begin{array}{l}\text { Study } \\
\text { design }\end{array}$} & \multirow{2}{*}{$\begin{array}{l}\text { Basic theories } \\
\text { of emotions }\end{array}$} & \multirow[b]{2}{*}{ Measures } & \multirow{2}{*}{$\begin{array}{l}\text { Rated } \\
\text { Qual. }\end{array}$} \\
\hline & & & Age & Level & Culture & Sport & & & & & \\
\hline (11) Mella- & \multirow[t]{2}{*}{2008} & $N=12$ & $M_{\text {age }}=26.8$ & \multirow[t]{2}{*}{ Elite } & \multirow[t]{2}{*}{ English } & \multirow{2}{*}{$\begin{array}{l}\text { Rugby } \\
\text { union }\end{array}$} & \multirow{2}{*}{$\begin{array}{l}\text { Determination of } \\
\text { emotional experi- } \\
\text { ence in precompeti- } \\
\text { tive period }\end{array}$} & \multirow[t]{2}{*}{ Descriptive } & \multirow{2}{*}{$\begin{array}{l}\text { Time-to-event } \\
\text { paradigm }\end{array}$} & \multirow[t]{2}{*}{ Interv. } & \multirow[t]{2}{*}{12} \\
\hline $\begin{array}{l}\text { lieu, Hanton } \\
\text { \& Shearer }\end{array}$ & & $\mathrm{n}_{\mathrm{s}}=12$ & $S D=3.2$ & & & & & & & & \\
\hline \multirow{3}{*}{$\begin{array}{l}\text { (12) Verger } \\
\text { \& Bertrank }\end{array}$} & \multirow[t]{3}{*}{2008} & \multirow{3}{*}{$\begin{array}{l}N=6 \\
\mathrm{n}_{\mathrm{s}}=6\end{array}$} & $M_{\mathrm{age}}=$ & \multirow{3}{*}{$\begin{array}{l}\text { Non } \\
\text { elite }\end{array}$} & \multirow[t]{3}{*}{ French } & \multirow{3}{*}{$\begin{array}{l}\text { Rugby } \\
\text { union }\end{array}$} & \multirow{3}{*}{$\begin{array}{l}\text { Routineacquisition } \\
\text { with goal kickers }\end{array}$} & \multirow[t]{3}{*}{ Descriptive } & \multirow[t]{3}{*}{ l } & \multirow{3}{*}{$\begin{array}{l}\text { Interv.\& } \\
\text { obj. charact. } \\
\text { (Video) }\end{array}$} & \multirow[t]{3}{*}{7} \\
\hline & & & 18.64 & & & & & & & & \\
\hline & & & $S D=1.79$ & & & & & & & & \\
\hline \multirow{2}{*}{$\begin{array}{l}\text { (13) Hagtvet } \\
\text { \&Hanin }\end{array}$} & \multirow[t]{2}{*}{2008} & $N=12$ & $M_{\text {agestudy } 1}$ & \multirow[t]{2}{*}{ Elite } & \multirow[t]{2}{*}{ Finnish } & \multirow{2}{*}{$\begin{array}{l}\text { Ice } \\
\text { hockey }\end{array}$} & \multirow{2}{*}{$\begin{array}{l}\text { Application of } \\
\text { IZOF model on ice } \\
\text { hockeypopulation } \\
\text { and the relationship } \\
\text { between best and } \\
\text { worst performances }\end{array}$} & \multirow[t]{2}{*}{ Descriptive } & \multirow[t]{2}{*}{ IZOF model } & Intensity & 13 \\
\hline & & $\mathrm{n}_{\mathrm{s}}=12$ & $\begin{array}{l}=\text { ages } \\
\text { ranged } \\
\text { from18-32 } \\
\text { yrs. }\end{array}$ & & & & & & & $\begin{array}{l}\text { scale applied } \\
\text { to a list of } \\
\text { emotions }\end{array}$ & \\
\hline (14) Nicholls & 2007 & $N=11$ & $M_{\text {age }}=17.9$ & Elite & English & Rugby & Examination of the & Descriptive & Lazarus-CMR & Daily-diaries & 9 \\
\hline \& Polman & & $\mathrm{n}_{\mathrm{s}}=11$ & $S D=0.3$ & & & & $\begin{array}{l}\text { stressors, copıng } \\
\text { strategies and } \\
\text { coping effectiveness }\end{array}$ & & & & \\
\hline (15) Polman, & 2007 & $N=12$ & $M_{\mathrm{age}}=26.5$ & Elite & English & Rugby & Examination & Observa- & Home advan- & Daily-diaries & 9 \\
\hline $\begin{array}{l}\text { Nicholls, } \\
\text { Cohen \& } \\
\text { Borkoles }\end{array}$ & & $\mathrm{n}_{\mathrm{s}}=12$ & $S D=4.3$ & & & le & $\begin{array}{l}\text { of relationships } \\
\text { between mood and } \\
\text { game location }\end{array}$ & & $\begin{array}{l}\text { tage approach } \\
\& \text { mood states } \\
\text { model }\end{array}$ & & \\
\hline
\end{tabular}


Table 1 (continued)

\begin{tabular}{|c|c|c|c|c|c|c|c|c|c|c|c|}
\hline \multirow[b]{2}{*}{ Authors } & \multirow[b]{2}{*}{ Year } & \multirow{2}{*}{$\begin{array}{l}\text { Sample } \\
\text { size }\end{array}$} & \multicolumn{4}{|c|}{ Sample characteristics } & \multirow[b]{2}{*}{ Study question } & \multirow{2}{*}{$\begin{array}{l}\text { Study } \\
\text { design }\end{array}$} & \multirow{2}{*}{$\begin{array}{l}\text { Basic theories } \\
\text { of emotions }\end{array}$} & \multirow[b]{2}{*}{ Measures } & \multirow{2}{*}{$\begin{array}{l}\text { Rated } \\
\text { Qual. }\end{array}$} \\
\hline & & & Age & Level & Culture & Sport & & & & & \\
\hline \multirow{5}{*}{$\begin{array}{l}\text { (16) Robazza } \\
\text { \& Bortoli }\end{array}$} & \multirow[t]{5}{*}{2007} & $N=197$ & elite $M_{\text {age }}=$ & \multirow{5}{*}{$\begin{array}{l}\text { Elite } \\
\& \text { non } \\
\text { elite }\end{array}$} & \multirow{5}{*}{ Italian } & \multirow{5}{*}{$\begin{array}{l}\text { Rugby } \\
\text { union }\end{array}$} & \multirow{5}{*}{$\begin{array}{l}\text { Extension of the } \\
\text { direction concept } \\
\text { to anger }\end{array}$} & \multirow{5}{*}{$\begin{array}{l}\text { Observa- } \\
\text { tional }\end{array}$} & \multirow{5}{*}{$\begin{array}{l}\text { Directional } \\
\text { approach }\end{array}$} & \multirow[t]{5}{*}{ Quest. } & \multirow[t]{5}{*}{17} \\
\hline & & $n=197$ & $\begin{array}{l}26.6 \\
\text { elite } S D= \\
3.89\end{array}$ & & & & & & & & \\
\hline & & & non elite & & & & & & & & \\
\hline & & & $\begin{array}{l}M_{\text {age }}= \\
26.23\end{array}$ & & & & & & & & \\
\hline & & & $\begin{array}{l}\text { non elite } \\
S D=4.7\end{array}$ & & & & & & & & \\
\hline \multirow{2}{*}{$\begin{array}{l}\text { (17) Uphill \& } \\
\text { Jones }\end{array}$} & \multirow[t]{2}{*}{2007} & $N=12$ & $M_{\text {age }}=27.0$ & \multirow[t]{2}{*}{ Elite } & \multirow[t]{2}{*}{ Und. } & \multirow{2}{*}{$\begin{array}{l}\text { Rugby } \\
\text { union }\end{array}$} & \multirow{2}{*}{$\begin{array}{l}\text { Examination of the } \\
\text { emotional experi- } \\
\text { ence with the CMR } \\
\text { ineory }\end{array}$} & \multirow[t]{2}{*}{ Descriptive } & \multirow[t]{2}{*}{ Lazarus-CMR } & \multirow[t]{2}{*}{ Interv. } & \multirow[t]{2}{*}{12} \\
\hline & & $\mathrm{n}_{\mathrm{s}}=3$ & $S D=6.03$ & & & & & & & & \\
\hline \multirow{3}{*}{$\begin{array}{l}\text { (18) Dunn, } \\
\text { Gotwals, } \\
\text { Causgrove } \\
\text { Dunn, \& } \\
\text { syrotuık }\end{array}$} & \multirow[t]{3}{*}{2006} & $N=138$ & $M_{\mathrm{age}}=$ & \multirow[t]{3}{*}{ Elite } & \multirow[t]{3}{*}{ Canadian } & \multirow{3}{*}{$\begin{array}{l}\text { Canadian } \\
\text { football }\end{array}$} & \multirow{3}{*}{$\begin{array}{l}\text { Examination of } \\
\text { the relationship } \\
\text { between perfec- } \\
\text { tionism and anger } \\
\text { aısposıtions }\end{array}$} & \multirow{3}{*}{$\begin{array}{l}\text { Observa- } \\
\text { tional }\end{array}$} & Perfectionism \& & Quest. & 9 \\
\hline & & $n_{s}=138$ & 1827 & & & & & & anger & & \\
\hline & & & $S D=.71$ & & & & & & & & \\
\hline (19) Neil, & 2006 & $N=115$ & $M_{\mathrm{age}}=$ & Elite & English & Rugby & Comparison of & Observa- & Directional & Quest. & 17 \\
\hline Mellalieu \& & & $n-115$ & & \& non & & union & intensity and direc- & tional & approach & & \\
\hline $\mathrm{Ha}$ & & & $S D=2.92$ & & & & $\begin{array}{l}\text { tion of anxiety with } \\
\text { usage of mental } \\
\text { skills }\end{array}$ & & & & \\
\hline
\end{tabular}




\begin{tabular}{|c|c|c|c|c|c|c|c|c|c|c|c|}
\hline \multirow[b]{2}{*}{ Authors } & \multirow[b]{2}{*}{ Year } & \multirow{2}{*}{$\begin{array}{l}\text { Sample } \\
\text { size }\end{array}$} & \multicolumn{4}{|c|}{ Sample characteristics } & \multirow[b]{2}{*}{ Study question } & \multirow{2}{*}{$\begin{array}{l}\text { Study } \\
\text { design }\end{array}$} & \multirow{2}{*}{$\begin{array}{c}\text { Basic theories } \\
\text { of emotions }\end{array}$} & \multirow[b]{2}{*}{ Measures } & \multirow{2}{*}{$\begin{array}{l}\text { Rated } \\
\text { Qual. }\end{array}$} \\
\hline & & & Age & Level & Culture & Sport & & & & & \\
\hline \multirow{2}{*}{$\begin{array}{l}\text { (20) Nicholls, } \\
\text { Holt, Polman } \\
\text { \& Bloomfield }\end{array}$} & \multirow[t]{2}{*}{2006} & $N=8$ & $M_{\text {age }}=24.6$ & \multirow[t]{2}{*}{ Elite } & \multirow{2}{*}{$\begin{array}{l}\text { Multicult. } \\
\text { Caucasian }\end{array}$} & \multirow{2}{*}{$\begin{array}{l}\text { Rugby } \\
\text { union }\end{array}$} & \multirow{2}{*}{$\begin{array}{l}\text { Examination of } \\
\text { stressors, coping } \\
\text { strategies and } \\
\text { coping effective- } \\
\text { ness }\end{array}$} & \multirow[t]{2}{*}{ Descriptive } & \multirow[t]{2}{*}{ Lazarus-CMR } & \multirow{2}{*}{$\begin{array}{l}\text { Daily-diaries } \\
\text { sheets }\end{array}$} & \multirow[t]{2}{*}{9} \\
\hline & & $\mathrm{n}_{\mathrm{s}}=8$ & $S D=2.2$ & & & & & & & & \\
\hline \multirow{7}{*}{$\begin{array}{l}\text { (21) Roba- } \\
\text { zza, Bertollo } \\
\text { \& Bortoli }\end{array}$} & \multirow[t]{7}{*}{2006} & $N=100$ & elite $M_{\text {age }}=$ & \multirow{7}{*}{$\begin{array}{l}\text { Elite } \\
\& \text { non } \\
\text { elite }\end{array}$} & \multirow[t]{7}{*}{ Italian } & \multirow{7}{*}{$\begin{array}{l}\text { Rugby } \\
\text { union }\end{array}$} & \multirow{7}{*}{$\begin{array}{l}\text { Comparison of fre- } \\
\text { quency and direc- } \\
\text { tion of competitive } \\
\text { anger }\end{array}$} & \multirow[t]{7}{*}{ Descriptive } & \multirow{7}{*}{$\begin{array}{l}\text { Directional } \\
\text { approach }\end{array}$} & \multirow[t]{7}{*}{ Quest. } & \multirow[t]{7}{*}{14} \\
\hline & & $\mathrm{n}_{\mathrm{s}}=50$ & & & & & & & & & \\
\hline & & & $\begin{array}{l}\text { elite } S D= \\
4.2\end{array}$ & & & & & & & & \\
\hline & & & non elite & & & & & & & & \\
\hline & & & $M_{\text {age }}=$ & & & & & & & & \\
\hline & & & 24.64 & & & & & & & & \\
\hline & & & $\begin{array}{l}\text { Non elite } \\
S D=4.24\end{array}$ & & & & & & & & \\
\hline \multirow{3}{*}{$\begin{array}{l}\text { (22) Valance, } \\
\text { Dunn \& } \\
\text { Causgrove } \\
\text { Dunn }\end{array}$} & \multirow[t]{3}{*}{2006} & $N=229$ & $M_{\text {age }}=$ & \multirow[t]{3}{*}{ Elite } & \multirow[t]{3}{*}{ Canadian } & \multirow{3}{*}{$\begin{array}{l}\text { Ice } \\
\text { hockey }\end{array}$} & \multirow{3}{*}{$\begin{array}{l}\text { Examination of } \\
\text { the relationship } \\
\text { between anger and } \\
\text { perfectionism }\end{array}$} & Observa- & Interaction & Quest. & 11 \\
\hline & & $\mathrm{n}_{\mathrm{s}}=229$ & 14.15 & & & & & tional & paradigm & & \\
\hline & & & $S D=1.03$ & & & & & & & & \\
\hline (23) Jones, & 2005 & $N=21$ & Und. & Elite & English & Rugby & Examination of & Observa- & HomeAdvan- & Objective & 17 \\
\hline Bray \& & & $\mathrm{n}_{\mathrm{s}}=21$ & & & & league & the relationship & tional & tage approach & charact. & \\
\hline Uivier & & & & & & & location and & & & (viaeo) & \\
\hline
\end{tabular}


Table 1 (continued)

\begin{tabular}{|c|c|c|c|c|c|c|c|c|c|c|c|}
\hline \multirow[b]{2}{*}{ Authors } & \multirow[b]{2}{*}{ Year } & \multirow{2}{*}{$\begin{array}{l}\text { Sample } \\
\text { size }\end{array}$} & \multicolumn{4}{|c|}{ Sample characteristics } & \multirow[b]{2}{*}{ Study question } & \multirow{2}{*}{$\begin{array}{l}\text { Study } \\
\text { design }\end{array}$} & \multirow{2}{*}{$\begin{array}{c}\text { Basic theories } \\
\text { of emotions }\end{array}$} & \multirow[b]{2}{*}{ Measures } & \multirow{2}{*}{$\begin{array}{l}\text { Rated } \\
\text { Qual. }\end{array}$} \\
\hline & & & Age & Level & Culture & Sport & & & & & \\
\hline $\begin{array}{l}\text { (24) Lons- } \\
\text { dale \&Howe }\end{array}$ & 2004 & $\begin{array}{l}N=107 \mathrm{n}_{\mathrm{s}} \\
=107\end{array}$ & $\begin{array}{l}M_{\mathrm{age}}=22.6 \\
S D=3.1\end{array}$ & $\begin{array}{l}\text { Non } \\
\text { elite }\end{array}$ & Canadian & $\begin{array}{l}\text { Rugby } \\
\text { union }\end{array}$ & $\begin{array}{l}\text { Examination of } \\
\text { stressors and } \\
\text { appraisal of rugby } \\
\text { union players } \\
\text { during sport season }\end{array}$ & Descriptive & Lazarus-CMR & Quest. & 10 \\
\hline $\begin{array}{l}\text { (25) Eys, } \\
\text { Hardy, } \\
\text { Carron \& } \\
\text { Beauchamp }\end{array}$ & 2003 & $\begin{array}{l}N=392 \\
\mathrm{n}_{\mathrm{s}}=170\end{array}$ & $\begin{array}{l}M_{\text {age }}= \\
17.12 \\
S D=3.76\end{array}$ & $\begin{array}{l}\text { Elite } \\
\text { \& non } \\
\text { elite }\end{array}$ & Und. & $\begin{array}{l}\text { Rugby } \\
\text { union }\end{array}$ & $\begin{array}{l}\text { Relationship } \\
\text { between Task } \\
\text { cohesionand pre- } \\
\text { competitive anxiety } \\
\text { state }\end{array}$ & $\begin{array}{l}\text { Observa- } \\
\text { tional }\end{array}$ & $\begin{array}{l}\text { Cohesion and } \\
\text { Directional } \\
\text { approach }\end{array}$ & Quest. & 13 \\
\hline $\begin{array}{l}\text { (26) Golby, } \\
\text { Sheard \& } \\
\text { Lavalee }\end{array}$ & 2003 & $\begin{array}{l}N=70 \\
\mathrm{n}_{\mathrm{s}}=70\end{array}$ & $\begin{array}{l}M_{\text {age }}=25.5 \\
S D=3.2\end{array}$ & $\begin{array}{l}\text { Non } \\
\text { elite }\end{array}$ & $\begin{array}{l}\text { Multicult. } \\
\text { European }\end{array}$ & $\begin{array}{l}\text { Rugby } \\
\text { league }\end{array}$ & $\begin{array}{l}\text { Analysis of mental } \\
\text { toughness in } \\
\text { national rugby } \\
\text { league football } \\
\text { teams }\end{array}$ & $\begin{array}{l}\text { Observa- } \\
\text { tional }\end{array}$ & $\begin{array}{l}\text { Mental tough- } \\
\text { ness approach }\end{array}$ & Quest. & 17 \\
\hline (27) Jackson & 2003 & $\begin{array}{l}N=20 \\
\mathrm{n}_{\mathrm{s}}=20\end{array}$ & $\begin{array}{l}M_{\mathrm{age}}=26.4 \\
S D=3.3\end{array}$ & Elite & $\begin{array}{l}\text { Multicult. } \\
\text { Internat. }\end{array}$ & $\begin{array}{l}\text { Rugby } \\
\text { union }\end{array}$ & $\begin{array}{l}\text { Analysis of preper- } \\
\text { formance routine } \\
\text { consistency }\end{array}$ & Descriptive & l & $\begin{array}{l}\text { Obj. charact. } \\
\text { (Video) }\end{array}$ & 11 \\
\hline $\begin{array}{l}\text { (28) Dunn \& } \\
\text { Syrotuik }\end{array}$ & 2003 & $\begin{array}{l}N=170 \\
\mathrm{n}_{\mathrm{s}}=170\end{array}$ & $\begin{array}{l}M_{\mathrm{age}}= \\
18.24 \\
S D=0.66\end{array}$ & Elite & Canadian & $\begin{array}{l}\text { Canadian } \\
\text { Football }\end{array}$ & $\begin{array}{l}\text { Investigation of } \\
\text { worry in Canadian } \\
\text { football }\end{array}$ & $\begin{array}{l}\text { Observa- } \\
\text { tional }\end{array}$ & $\begin{array}{l}\text { Mutidimen- } \\
\text { sional anxiety }\end{array}$ & Quest. & 8 \\
\hline
\end{tabular}




\begin{tabular}{|c|c|c|c|c|c|c|c|c|c|c|c|}
\hline \multirow[b]{2}{*}{ Authors } & \multirow[b]{2}{*}{ Year } & \multirow{2}{*}{$\begin{array}{l}\text { Sample } \\
\text { size }\end{array}$} & \multicolumn{4}{|c|}{ Sample characteristics } & \multirow[b]{2}{*}{ Study question } & \multirow{2}{*}{$\begin{array}{l}\text { Study } \\
\text { design }\end{array}$} & \multirow{2}{*}{$\begin{array}{c}\text { Basic theories } \\
\text { of emotions }\end{array}$} & \multirow[b]{2}{*}{ Measures } & \multirow{2}{*}{$\begin{array}{l}\text { Rated } \\
\text { Qual. }\end{array}$} \\
\hline & & & Age & Level & Culture & Sport & & & & & \\
\hline \multirow{2}{*}{$\begin{array}{l}\text { (29) Golby \& } \\
\text { Sheard }\end{array}$} & \multirow[t]{2}{*}{2003} & $N=115$ & $M_{\text {age }}=25.5$ & \multirow[t]{2}{*}{ Elite } & \multirow[t]{2}{*}{ Und. } & \multirow{2}{*}{$\begin{array}{l}\text { Rugby } \\
\text { league }\end{array}$} & \multirow{2}{*}{$\begin{array}{l}\text { Examination of } \\
\text { the relationship } \\
\text { between mental } \\
\text { toughness and har- } \\
\text { dinessatdifferent } \\
\text { levels of practice }\end{array}$} & \multirow{2}{*}{$\begin{array}{l}\text { Observa- } \\
\text { tional }\end{array}$} & \multirow{2}{*}{$\begin{array}{l}\text { Cultural } \\
\text { approach, } \\
\text { mental tough- } \\
\text { ness \& hardi- } \\
\text { ness approaches }\end{array}$} & \multirow[t]{2}{*}{ Quest. } & \multirow[t]{2}{*}{9} \\
\hline & & $\mathrm{n}_{\mathrm{s}}=115$ & $S D=3.3$ & & & & & & & & \\
\hline \multirow{2}{*}{$\begin{array}{l}\text { (30) Noblet \& } \\
\text { Gifford }\end{array}$} & \multirow[t]{2}{*}{2002} & $N=32$ & Und. & \multirow[t]{2}{*}{ Elite } & \multirow[t]{2}{*}{ Australian } & \multirow{2}{*}{$\begin{array}{l}\text { Australian } \\
\text { Football }\end{array}$} & \multirow{2}{*}{$\begin{array}{l}\text { Examination of the } \\
\text { sources of stress } \\
\text { in professional } \\
\text { Australian football } \\
\text { practice }\end{array}$} & \multirow[t]{2}{*}{ Descriptive } & \multirow[t]{2}{*}{ l } & \multirow{2}{*}{$\begin{array}{l}\text { Focus group } \\
\text { \& Interv. }\end{array}$} & \multirow[t]{2}{*}{7} \\
\hline & & $\mathrm{n}_{\mathrm{s}}=32$ & & & & & & & & & \\
\hline \multirow{2}{*}{$\begin{array}{l}\text { (31) D’Urso, } \\
\text { Petrosso \& } \\
\text { Robazza }\end{array}$} & \multirow[t]{2}{*}{2002} & $N=33$ & $M_{\text {age }}=$ & \multirow[t]{2}{*}{ Elite } & \multirow[t]{2}{*}{ Italian } & \multirow{2}{*}{$\begin{array}{l}\text { Rugby } \\
\text { union }\end{array}$} & \multirow{2}{*}{$\begin{array}{l}\text { Application of } \\
\text { IZOF model on } \\
\text { rugby population } \\
\text { and the relationship } \\
\text { between pre-and } \\
\text { per-competitive } \\
\text { period }\end{array}$} & \multirow{2}{*}{$\begin{array}{l}\text { Observa- } \\
\text { tional }\end{array}$} & \multirow[t]{2}{*}{ IZOF model } & Interv.\& & 13 \\
\hline & & $\mathrm{n}_{\mathrm{s}}=33$ & $\begin{array}{l}27.0 \\
S D=4.5\end{array}$ & & & & & & & & \\
\hline (32) Anshel & 2001 & $N=28$ & $M_{\mathrm{age}}=22.4$ & Und. & Australian & Rugby & Qualitative valida- & Descriptive & Lazarus-CMR & Interv. & 13 \\
\hline & & $\mathrm{n}_{\mathrm{s}}=28$ & $\begin{array}{l}S D=\text { from } \\
18.2 \text { to } \\
27.2 \text { yrs. }\end{array}$ & & & lea & $\begin{array}{l}\text { tion of a model for } \\
\text { coping with acute } \\
\text { stress in sport }\end{array}$ & & & & \\
\hline (33) Hanton, & 2000 & $N=100$ & $M_{\text {age }}=23.4$ & Elite & Und. & Rugby & Examination of & Observa- & Directional & Quest. & 17 \\
\hline $\begin{array}{l}\text { Jones \& } \\
\text { Mullen }\end{array}$ & & $\mathrm{n}_{\mathrm{s}}=50$ & $S D=3.4$ & $\begin{array}{l}\& \text { non } \\
\text { elite }\end{array}$ & & league & $\begin{array}{l}\text { the relationship } \\
\text { between anxiety } \\
\text { and the type of } \\
\text { sport }\end{array}$ & $t$ & 2 & & ntinued) \\
\hline
\end{tabular}




\begin{tabular}{|c|c|c|c|c|c|c|c|c|c|c|c|}
\hline \multirow[b]{2}{*}{ Authors } & \multirow[b]{2}{*}{ Year } & \multirow{2}{*}{$\begin{array}{l}\text { Sample } \\
\text { size }\end{array}$} & \multicolumn{4}{|c|}{ Sample characteristics } & \multirow[b]{2}{*}{ Study question } & \multirow{2}{*}{$\begin{array}{l}\text { Study } \\
\text { design }\end{array}$} & \multirow{2}{*}{$\begin{array}{c}\text { Basic theories } \\
\text { of emotions }\end{array}$} & \multirow[b]{2}{*}{ Measures } & \multirow{2}{*}{$\begin{array}{l}\text { Rated } \\
\text { Qual. }\end{array}$} \\
\hline & & & Age & Level & Culture & Sport & & & & & \\
\hline \multirow{2}{*}{$\begin{array}{l}\text { (34) Terry, } \\
\text { Carron, Pink, } \\
\text { Lane, Jones \& } \\
\text { Hall }\end{array}$} & \multirow[t]{2}{*}{2000} & & $M_{\mathrm{age}}=25.9$ & \multirow[t]{2}{*}{ Und. } & \multirow[t]{2}{*}{ English } & \multirow{2}{*}{$\begin{array}{l}\text { Rugby } \\
\text { union }\end{array}$} & \multirow{2}{*}{$\begin{array}{l}\text { Examination of the } \\
\text { effects of percep- } \\
\text { tion of group cohe- } \\
\text { sion on mood }\end{array}$} & \multirow{2}{*}{$\begin{array}{l}\text { Observa- } \\
\text { tional }\end{array}$} & \multirow{2}{*}{$\begin{array}{l}\text { Cohesion and } \\
\text { mood states } \\
\text { model }\end{array}$} & \multirow[t]{2}{*}{ Quest. } & \multirow[t]{2}{*}{13} \\
\hline & & $\mathrm{n}_{\mathrm{s}}=173$ & $S D=5.9$ & & & & & & & & \\
\hline \multirow[t]{3}{*}{ (35) Dunn } & \multirow[t]{3}{*}{1999} & $N=178$ & $M_{\text {age }}=$ & \multirow[t]{3}{*}{ Univ. } & \multirow[t]{3}{*}{ Canadian } & \multirow{3}{*}{$\begin{array}{l}\text { Ice } \\
\text { Hockey }\end{array}$} & \multirow{3}{*}{$\begin{array}{l}\text { Examination of } \\
\text { competitive worry } \\
\text { in ice hockey. }\end{array}$} & \multirow[t]{3}{*}{ Descriptive } & \multirow{3}{*}{$\begin{array}{l}\text { Multidimen- } \\
\text { sional anxiety }\end{array}$} & \multirow[t]{3}{*}{ Quest. } & \multirow[t]{3}{*}{8} \\
\hline & & $\mathrm{n}_{\mathrm{s}}=178$ & & & & & & & & & \\
\hline & & & $S D=1.62$ & & & & & & & & \\
\hline \multirow{2}{*}{$\begin{array}{l}\text { (36) Green- } \\
\text { lees, Nunn, } \\
\text { Graydon \& } \\
\text { Maynard }\end{array}$} & \multirow[t]{2}{*}{1999} & $N=66$ & $M_{\mathrm{age}}=2.8$ & \multirow{2}{*}{$\begin{array}{l}\text { Non } \\
\text { elite }\end{array}$} & \multirow[t]{2}{*}{ Und. } & \multirow{2}{*}{$\begin{array}{l}\text { Rugby } \\
\text { union }\end{array}$} & \multirow{2}{*}{$\begin{array}{l}\text { Examination of } \\
\text { the relationship } \\
\text { between collective } \\
\text { efficacy and pre- } \\
\text { competitive affects }\end{array}$} & \multirow{2}{*}{$\begin{array}{l}\text { Observa- } \\
\text { tional }\end{array}$} & Positive- & Quest. & 11 \\
\hline & & $\mathrm{n}_{\mathrm{s}}=66$ & $S D=6.3$ & & & & & & $\begin{array}{l}\text { Negative affect } \\
\text { approach }\end{array}$ & & \\
\hline (37) Wilson & 1999 & $N=18$ & $M_{\mathrm{age}}=28.9$ & Elite & German & Rugby & Examination of & Observa- & Reversal Theory & Quest. & 13 \\
\hline \& Kherr & & $\mathrm{n}_{\mathrm{s}}=18$ & $S D=2.64$ & & & union & $\begin{array}{l}\text { aftective responses } \\
\text { to success and } \\
\text { failure }\end{array}$ & & & & \\
\hline (38) Bush- & 1998 & $N=91$ & Und. & Non & American & Ice & & Observa- & Aggressivity & Quest. & 9 \\
\hline man \&wells & & $\mathrm{n}_{\mathrm{s}}=91$ & & & & key & $\begin{array}{l}\text { the relationship } \\
\text { between aggres- } \\
\text { siveness and penal- } \\
\text { tiesinice hockey }\end{array}$ & & ap & & \\
\hline (39) Terry, & 1998 & $N=100$ & $M_{\mathrm{age}}=24.1$ & Und. & Und. & Rugby & Examination of & Observa- & Home advan- & Quest. & 13 \\
\hline $\begin{array}{l}\text { Walrond \& } \\
\text { Carron }\end{array}$ & & $\mathrm{n}_{\mathrm{s}}=100$ & $S D=3.9$ & & & & $\begin{array}{l}\text { the influence of } \\
\text { the game location } \\
\text { on athletes' mood } \\
\text { states }\end{array}$ & & $\begin{array}{l}\text { tage model\& } \\
\text { mood states } \\
\text { model }\end{array}$ & & \\
\hline
\end{tabular}




\begin{tabular}{|c|c|c|c|c|c|c|c|c|c|c|c|}
\hline \multirow[b]{2}{*}{ Authors } & \multirow[b]{2}{*}{ Year } & \multirow{2}{*}{$\begin{array}{l}\text { Sample } \\
\text { size }\end{array}$} & \multicolumn{4}{|c|}{ Sample characteristics } & \multirow[b]{2}{*}{ Study question } & \multirow{2}{*}{$\begin{array}{l}\text { Study } \\
\text { design }\end{array}$} & \multirow{2}{*}{$\begin{array}{c}\text { Basic theories } \\
\text { of emotions }\end{array}$} & \multirow[b]{2}{*}{ Measures } & \multirow{2}{*}{$\begin{array}{l}\text { Rated } \\
\text { Qual. }\end{array}$} \\
\hline & & & Age & Level & Culture & Sport & & & & & \\
\hline \multirow{2}{*}{$\begin{array}{l}\text { (40) Lane, } \\
\text { Rodger \& } \\
\text { Karageorgis }\end{array}$} & \multirow[t]{2}{*}{1997} & $N=86$ & $M_{\text {age }}=23.7$ & \multirow{2}{*}{$\begin{array}{l}\text { Non } \\
\text { elite }\end{array}$} & \multirow[t]{2}{*}{ English } & \multirow{2}{*}{$\begin{array}{l}\text { Rugby } \\
\text { league }\end{array}$} & \multirow{2}{*}{$\begin{array}{l}\text { Examination of } \\
\text { antecedents of } \\
\text { anxiety state in } \\
\text { rugby }\end{array}$} & \multirow{2}{*}{$\begin{array}{l}\text { Observa- } \\
\text { tional }\end{array}$} & \multirow{2}{*}{$\begin{array}{l}\text { Cognitive- } \\
\text { somatic anxiety } \\
\text { approach }\end{array}$} & \multirow[t]{2}{*}{ Quest. } & \multirow[t]{2}{*}{13} \\
\hline & & $\mathrm{n}_{\mathrm{s}}=86$ & $S D=4.8$ & & & & & & & & \\
\hline \multirow{2}{*}{$\begin{array}{l}\text { (41) Hanin \& } \\
\text { Syrjä }\end{array}$} & \multirow{2}{*}{1995} & $N=46$ & $M_{\text {age }}=16.3$ & \multirow[t]{2}{*}{ Elite } & \multirow[t]{2}{*}{ Finnish } & \multirow{2}{*}{$\begin{array}{l}\text { Ice } \\
\text { Hockey }\end{array}$} & \multirow{2}{*}{$\begin{array}{l}\text { Application of the } \\
\text { IZOF model to } \\
\text { positive and nega- } \\
\text { tive affects in ice } \\
\text { hockey }\end{array}$} & \multirow[t]{2}{*}{ Descriptive } & \multirow[t]{2}{*}{ IZOF model } & \multirow{2}{*}{$\begin{array}{l}\text { Quest. \& } \\
\text { scales }\end{array}$} & \multirow[t]{2}{*}{10} \\
\hline & & $\mathrm{n}_{\mathrm{s}}=46$ & $S D=0.6$ & & & & & & & & \\
\hline \multirow{2}{*}{$\begin{array}{l}\text { (42) Kerr \& } \\
\text { Schaik }\end{array}$} & \multirow[t]{2}{*}{1995} & $N=17$ & $M_{\text {age }}=29.5$ & \multirow[t]{2}{*}{ Elite } & \multirow[t]{2}{*}{ German } & \multirow{2}{*}{$\begin{array}{l}\text { Rugby } \\
\text { union }\end{array}$} & \multirow{2}{*}{$\begin{array}{l}\text { Examination of the } \\
\text { effects of the game } \\
\text { locationand the } \\
\text { outcome on psy- } \\
\text { chological mood } \\
\text { state }\end{array}$} & \multirow{2}{*}{$\begin{array}{l}\text { Observa- } \\
\text { tional }\end{array}$} & \multirow{2}{*}{$\begin{array}{l}\text { Home advan- } \\
\text { tage approach }\end{array}$} & \multirow[t]{2}{*}{ Quest. } & 9 \\
\hline & & $\mathrm{n}_{\mathrm{s}}=17$ & $S D=2.88$ & & & & & & & & \\
\hline $\begin{array}{l}\text { (43) Freud- } \\
\text { enberger \& } \\
\text { Bergandi }{ }^{1}\end{array}$ & 1994 & l & 1 & l & l & $\begin{array}{l}\text { US Foot- } \\
\text { ball }\end{array}$ & $\begin{array}{l}\text { Review of the liter- } \\
\text { ature dealing with } \\
\text { sport psychology in } \\
\text { American football }\end{array}$ & Review & l & l & l \\
\hline (44) Kherr \& & 1994 & $N=109$ & $M_{\mathrm{age}}=19.1$ & Non & Und. & Rugby & Relationship & Descriptive & Mood and anxi- & Quest. & 8 \\
\hline Svebak & & $\mathrm{n}_{\mathrm{s}}=35$ & $S D=$ Und & elite & & union & $\begin{array}{l}\text { between physical } \\
\text { practice and emo- } \\
\text { tional experience }\end{array}$ & & ies & & \\
\hline
\end{tabular}


Table 1 (continued)

\begin{tabular}{|c|c|c|c|c|c|c|c|c|c|c|c|}
\hline \multirow[b]{2}{*}{ Authors } & \multirow[b]{2}{*}{ Year } & \multirow{2}{*}{$\begin{array}{l}\text { Sample } \\
\text { size }\end{array}$} & \multicolumn{4}{|c|}{ Sample characteristics } & \multirow[b]{2}{*}{ Study question } & \multirow{2}{*}{$\begin{array}{l}\text { Study } \\
\text { design }\end{array}$} & \multirow{2}{*}{$\begin{array}{c}\text { Basic theories } \\
\text { of emotions }\end{array}$} & \multirow[b]{2}{*}{ Measures } & \multirow{2}{*}{$\begin{array}{l}\text { Rated } \\
\text { Qual. }\end{array}$} \\
\hline & & & Age & Level & Culture & Sport & & & & & \\
\hline \multirow{2}{*}{$\begin{array}{l}\text { (45) Dunn \& } \\
\text { Nielsen }\end{array}$} & \multirow[t]{2}{*}{1993} & $N=71$ & $M_{\text {age }}=22.0$ & \multirow[t]{2}{*}{ Univ. } & \multirow[t]{2}{*}{ Und. } & \multirow{2}{*}{$\begin{array}{l}\text { Ice } \\
\text { Hockey }\end{array}$} & \multirow{2}{*}{$\begin{array}{l}\text { Examination of the } \\
\text { cognitive dimen- } \\
\text { sions of threat } \\
\text { perception in ice } \\
\text { hockey and soccer }\end{array}$} & \multirow{2}{*}{$\begin{array}{l}\text { Preexperi- } \\
\text { mental }\end{array}$} & \multirow{2}{*}{$\begin{array}{l}\text { Cognitive } \\
\text { approach of } \\
\text { anxiety }\end{array}$} & \multirow[t]{2}{*}{ Quest. } & \multirow[t]{2}{*}{8} \\
\hline & & $\mathrm{n}_{\mathrm{s}}=46$ & $S D=$ Und & & & & & & & & \\
\hline \multirow{2}{*}{$\begin{array}{l}\text { (46)Jones \& } \\
\text { Swain }\end{array}$} & \multirow[t]{2}{*}{1992} & $N=69$ & $M_{\text {age }}=$ & \multirow{2}{*}{$\begin{array}{l}\text { Non } \\
\text { elite }\end{array}$} & \multirow[t]{2}{*}{ Und. } & \multirow{2}{*}{$\begin{array}{l}\text { Rugby } \\
\text { union }\end{array}$} & \multirow{2}{*}{$\begin{array}{l}\text { Relationship } \\
\text { between anxiety } \\
\text { direction and com- } \\
\text { petitiveness }\end{array}$} & \multirow{2}{*}{$\begin{array}{l}\text { Observa- } \\
\text { tional }\end{array}$} & \multirow{2}{*}{$\begin{array}{l}\text { Directional } \\
\text { approach }\end{array}$} & \multirow{2}{*}{$\begin{array}{l}\text { Quest. \& } \\
\text { scales }\end{array}$} & \multirow[t]{2}{*}{11} \\
\hline & & $n_{s}=24$ & $\begin{array}{l}20.93 \\
S D=2.55\end{array}$ & & & & & & & & \\
\hline \multirow{2}{*}{$\begin{array}{l}\text { (47) } \\
\text { McGowan \& } \\
\text { Shultz }\end{array}$} & \multirow[t]{2}{*}{1989} & $N=232$ & Und. & \multirow{2}{*}{$\begin{array}{l}\text { Non } \\
\text { elite }\end{array}$} & \multirow[t]{2}{*}{ Und. } & \multirow{2}{*}{$\begin{array}{l}\text { US Foot- } \\
\text { ball }\end{array}$} & \multirow{2}{*}{$\begin{array}{l}\text { Examination of } \\
\text { playing positions } \\
\text { on concentration } \\
\text { and mood states }\end{array}$} & \multirow{2}{*}{$\begin{array}{l}\text { Observa- } \\
\text { tional }\end{array}$} & \multirow{2}{*}{$\begin{array}{l}\text { Mood States } \\
\text { model }\end{array}$} & \multirow[t]{2}{*}{ Quest. } & 13 \\
\hline & & $n_{s}=232$ & & & & & & & & & \\
\hline (48) Maynard & 1987 & $N=22$ & $M_{\text {age }}=$ & Non & Und. & Rugby & Relationship & Observa- & Cognitive- & Quest. & 13 \\
\hline \& Howe & & $\mathrm{n}_{\mathrm{s}}=22$ & $\begin{array}{l}\text { from } 19 \text { to } \\
24 \text { yrs }\end{array}$ & elite & & union & $\begin{array}{l}\text { between anxiety } \\
\text { and performance }\end{array}$ & tional & $\begin{array}{l}\text { somatic anxiety } \\
\text { approach }\end{array}$ & & \\
\hline
\end{tabular}

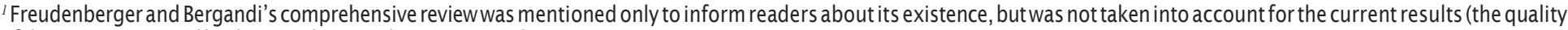
of the papers reviewed by these authors not being assessed).

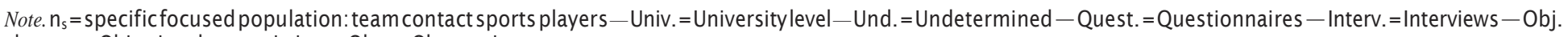
charact. $=$ Objective characteristics - Obs. $=$ Observation 


\title{
Table 2 Synthesis of the Main Reviewed Results
}

\author{
Studies Synthesis of the Main Results \\ 1 Aggressive behaviors seemed to be largely accepted by players as an integral part of the physicality of the Australian football game \\ (studied as an example of contact team-based sports for the authors) and were viewed as a competitive behavior within the game's \\ rule boundaries. \\ Players did not associate verbal aggression or "sledging" with an aggressive behavior. \\ Three types of aggressive behaviors were determined: anger (i.e., "hot" behavior), power (i.e., "cold" behavior) and thrill (i.e., "gratu- \\ itous" behavior) aggression. \\ Involvement in aggressive behaviors provoked some players' satisfaction and the introduction of severe punishments in the new rules \\ disappointed some interviewed players. \\ 2 Trajectories of negative and positive affective states were deflected by the need of satisfaction related to team selection (turning point \\ of the season). \\ 3 There was no difference in emotions and coping between u20, u1 8 and u 6 except higher trait anger for u20 players. \\ Young elite Hungarian players were characterized by a high level of self-confidence, coachability and curiosity, and a low level of \\ anxiety and coping skills. \\ $4 \quad$ A slight effect of the program was found with less aggressive acts and retaliations. \\ Players reported to play better and manage their emotions more effectively. \\ 5 Anger is significantly correlated to aggressiveness $(r=.71)$ and professionalization $(r=.22)$ \\ 6 The type of sport influenced the perception of the aggressiveness legitimacy. More specifically, considering rugby players studied in \\ this research, the players: \\ - Considered aggressiveness as more legitimate than athletes from individual sports and were more aggressive \\ - Presented a higher state anger than other athletes and tended to express it. \\ No significant difference between cultures was found.
}

$7 \quad$ The intervention allowed to influence significantly the players' interpretation of cognitive anxiety (more facilitating) and the players' self-confidence (i.,e., higher) and the coping efficiency. 
86 main stressors reported a higher mean frequency in training compared with matches: physical errors, mental errors, injuries, coach criticisms, weather and being confused by drills.

4 stressors had a higher mean frequency during matches compared with training sessions: observing an opponent cheating, receiving a wrong call from referees, opponents playing well, and being distracted by the crowd.

4 frequently used coping strategies were identified: increasing concentration, blocking, increased effort, and being focused on their own roles.

Anxiety was less frequent during matches but more intense than during training sessions.

Anger was more frequent and intense during matches compared with training sessions.

In general, higher intensities were reported during matches compared with training sessions.

Negative emotions were the most frequently cited emotions by players.

Coping effectiveness was significantly higher on training days than in matches.

A moderate negative correlation was found between emotional intensity and coping effectiveness.

Players experienced more stress on training days compared with match days.

Many stressors were "worse than normal" on the day of a match compared with the match day and the day before the match.

Players reported being "worse than normal" on 13 symptoms of stress on training days. Five symptoms were reported as worse than normal on match days and 8 on rest days.

Concerning the sources of life stress, the mean scores for diet, climate, sleep and home-life were significantly worse than normal and were significantly better than normal for home-life and friends.

Concerning the sources of stress during rest days, diet, sport and health were worse than normal, and home-life, friends and recreation were better than normal.

On training days, diet, climate, sleep, and health were reported as significantly worse than normal.

On match days, friends and sport training were significantly better than normal.

10 Anxiety and anger states were lower for adult players than for 418 players.

Adult players were abler to cope with anxiety in critical moments than 18 players. 


\section{Synthesis of the Main Results}

11 The level of anxiety state was stable from precompetitive time until event. However, the frequency of anxiety symptoms increased. Mental imagery was the most frequently used strategy by players to manage their arousal level and their self-confidence.

Players also frequently used self-talk and external verbal persuasion (e.g., from coaches, captain, etc.) to regulate their emotions. The main cognitive symptoms appeared 7 days to 2-3 hr before competitions, and then gave place to behavioral symptoms up to the beginning of the match.

12 To teach placed kick skills, the benefits of a constructivist approach were put forward, based on the process rather than on the result.

The five most reported stressors were physical errors, criticisms from the coach or a parent, mental errors, sustaining an injury and observing an opponent performing well.

The most reported coping strategies were blocking, increased effort, increased concentration, and taking advice.

Problem-focused coping strategies accounted for $66 \%$ of all reported coping strategies, emotion-focused coping strategies for $17.5 \%$ and avoidance coping strategies for $16.5 \%$.

The most frequently cited coping strategies were not rated as being the most effective responses but appeared to be related to the choice of the coping strategy deployed.

15 There were no significant differences between home and away matches according to moods, excepted for fatigue which was higher for away matches.

A significant relationship was found between moods and behaviors and subjective performance. These correlations were slightly different according to game locations.

The outcome of the match influenced the players' moods, and loosing largely decreased moods.

16 Players reported a facilitating interpretation of their anger state and thought that they controlled it. More specifically, anger at others was appraised as more facilitating than anger at oneself.

Cognitive and somatic anxiety was also appraised as facilitating.

Cognitive anxiety was a predictor of anger, and self-confidence was a predictor of anger control. 


\section{Table 2 (continued)}

\section{Synthesis of the Main Results}

17 The appraisal process was associated to the players' emotions.

Some different affects, which are not recognized by Lazarus, were put forward, but were quite similar to Lazarus's emotions (e.g., disappointment vs. sadness).

The core relational themes were not always similar to Lazarus's theory and the authors suggest that the environment might influence the appraisal process.

18 Anger state is correlated with the perceived coach pressure (.31), and concern over mistake (.35).

Anger state is correlated with Trait Anger (.35).

Maladaptive perfectionism predicted anger.

19 There was no difference between elite and nonelite players according to the intensity of anxiety state.

The elite players appraised anxiety as more facilitating than nonelite players.

Cognitive anxiety was viewed by players as handicapping their performance due to the fear associated to contact. Inversely, players appraised somatic anxiety as facilitating their performance because of their punctual nature, the associated increase effort sensation and the improvement of contact behaviors.

Elite players further used mental imagery and self-talk while nonelite players used more relaxation to manage their anxiety.

Players reported 24 different stressors with $44 \%$ represented by injuries, mental errors, and physical errors. These stressors fluctuated and declined as the season progressed.

Increasing concentration on the task was the most frequently cited coping strategy, followed by blocking, increasing effort and positive reappraisal.

Problem-focused coping strategy represented $70 \%$ of all coping strategies, emotion-focused coping strategies represented $14 \%$, and avoidance coping strategies represented $16 \%$.

Problem-focused and avoidance coping strategies decreased as the season progressed but emotion-focused coping strategies peaked in the middle of the season.

According to the most frequently cited coping strategies, only increased effort was evaluated as really effective.

Only 3 coping strategies (i.e., blocking, positive reappraisal and increasing effort) were used to deal with all three of the major stressors (i.e., injuries, mental errors and physical errors). 
Table 2 (continued)

\section{Synthesis of the Main Results}

21 Rugby players appraised their anger states as facilitating and reacted less to provocations.

22 Results found a positive relationship between high perfectionistic tendencies and anger responses, more particularly in situations that involved frustration and a negative evaluation.

Anger was more intensely experienced after a personal mistake in a high-criticality situation compared with a low-criticality situation.

23 There was no real influence of the game location on aggressive acts.

However, players seemed to be a little more aggressive when they were loosing during an away match.

There was no difference between both half-times concerning aggressive acts.

24 Being outplayed by the opposition and skill error events were rated as the greater stressors during the regular season and the playoff contexts.

During the preseason context, injuries and being outplayed by the opposition were rated as the most stressful event types.

Opposition aggression and environmental circumstances events were appraised as the least stressful event types.

Game context (i.e., preseason, regular season, and playoffs) influenced stress appraisal. Mean stress and challenge ratings significantly increased from preseason to playoffs.

Being outplayed by the opposition and game outcome events were rated as more challenging than stressful.

Interpersonal conflicts, poor refereeing decisions, team behaviors events and injuries were rated as more stressful than challenging.

Players who appraised their anxiety as facilitating, also positively appraised the task-cohesion.

26 Welsh players were more engaged and thought to had more control than the French.

Comparatively to the Irish players, the French appraised competitions more as a challenge than a threat.

Players presented a weak regulation skill of negative energy and mental visualization.

Players had a more mental toughness than the norm.

27 The cognitive time of the kickers' routine outlasted when players were under pressure according to the influence on the final outcome.

28 Players are mainlyworried in relation with the fear of failure, and the fear of negative social evaluation. The fears of injury and of the unknown also influence worry. 
Table 2 (continued)

\section{Synthesis of the Main Results}

29 International players presented a greater level of hardiness. They tended to appraise competitive situations as challenging rather than threatening, and they thought that they could largely influence the situation experienced.

30 Seventy-seven stressors were identified through 6 higher order themes: negative aspects of organizational systems and culture, worries about performance expectations and standards, career development concerns, negative aspects of interpersonal relationships, demanding nature of work itself, problems associated with work/non work interface.

31 Concerning affects, the IZOF profile did not discriminate performance.

Affects were modified during the competition due to internal behavior (e.g., individual counter-performance) and to external events (e.g., teammates' behaviors)

Low intensities of worry and inhibiting affects, and high scores of self-confidence, facilitating affects, psychological qualities, and physical and motor factors were appraised as facilitating performance.

32 Major stressors found were: "making a physical error" (32\%), "opponent cheating (21\%), "referee's decision" (16\%) "experiencing pain" (14\%), "opponent's success" (12\%) and "negative feedback from coach" (3\%).

$48 \%$ of all the players appraised situation as threatening, $34 \%$ as harming and $18 \%$ as challenging.

Players used every type of coping and the postcoping responses were more focused on cognitive activities (i.e., enhancement of attention, reappraisal).

33 Somatic and cognitive competitive anxiety was facilitating for rugby players.

A difference was found between rugby players and riffle shooters about the interpretation of somatic anxiety (more facilitating in rugby).

34 A difference between rugby, netball and rowing was found concerning mood and cohesion.

Rugby presented more group integration than the other sports studied, and more group attraction than rowing.

The perception of cohesion predicted the rugby players' mood responses (from $2.56 \%$ to $12.45 \%$ ).

For a rugby group, task cohesion and more specifically, attraction to the group, was inversely correlated to tension (-.19) and anger $(-.19)$.

For a rugby group, social cohesion, and more specifically, attraction to the group was inversely correlated to tension (.21), depression $(-.26)$, and fatigue (-.17), and positively correlated to vigor (.36). 


\section{Table 2 (continued)}

\section{Synthesis of the Main Results}

35 Competitive worry is influenced by the fear of failure, the fear of negative social evaluation, the fear of injury and support the notion of multiple worries.

36 Collective efficacy regarding team performance predicted positive affects and represented $22 \%$ of variance in the precompetitive positive affects.

Collective efficacy regarding match outcome predicted cognitive anxiety but accounted for $6.3 \%$ of the variance in cognitive anxiety intensities.

37 No difference was found according to somatic symptoms.

Level of tension decreased from pretime to posttime.

Anger and sullenness were lower, and relaxation and gratitude were higher for a positive outcome.

Resentment, sadness, humiliation were experienced when players had lost the game.

Affective changes were found associated with the outcome (i.e., winning produced pleasant emotional states and reduced stress; losing produced unpleasant emotional changes).

38 Penalized minutes for aggressive behaviors were correlated to the players' aggressiveness trait.

39 A slight home advantage was observed with the home teams winning $58.3 \%$ of the 12 games studied.

The mood iceberg profile was more pronounced before home games than before away games.

Home games were associated with significantly higher scores on vigor and self-confidence, and significantly lower scores on tension, depression, anger, fatigue, confusion, cognitive anxiety and somatic anxiety.

40 Perceived readiness (i.e., confidence about next outcome, mental readiness, physical readiness, rated performance in training, and rated performance in matches) accounted for $30.4 \%$ of variance in the expectations of positive performance, $17 \%$ for match conditions (i.e., weather and pitch conditions), and $15.9 \%$ for the coach's influence.

Perceived readiness and match conditions predicted self-confidence and somatic anxiety (respectively $30 \%$ and $11 \%$ of variance). 
41 Forty-four positive(PA) and 42 negative (NA) affects were experienced by players.

Concerning PA: $22.7 \%$ were facilitating, 11,4\% debilitating and $65.9 \%$ were both facilitating and debilitating.

Concerning NA: $17.9 \%$ were facilitating, $41 \%$ debilitating and $41 \%$ were both facilitating and debilitating.

$20.8 \%$ of affect experienced were facilitating and $25.3 \%$ debilitating.

Five PAwere debilitating and 7 NAwere facilitating. In otherwords, $30 \%$ of experienced affects had a reverse effecton the athletes' performance.

42 The game venue influenced arousal that was higher at home games than at away games.

No difference was found between home and away games according to precompetitive mood.

After winning, stress levels were significantly lower and arousal was significantly higher than after losing.

43 Note: this paper is a narrative review.

44 There was a significant increase of arousal in rugby compared with basketball and running.

Group mean scores for pleasant moods were approximately twice as many as those for unpleasant moods, but pleasant moods tended to decrease from pretime to posttime and especially in rugby.

Rugby provoked unpleasant moods (e.g., shame, humiliation, anger, and guilt) through external factors.

Unpleasant moods increased in rugby and more particularly, anger and sullenness.

Different appraisals for a same threatening situation was explained by the influence of the type of sport or the nature of the game.

No difference was found between high competitive and low competitive groups according to anxiety state.

High competitive group had more self-confidence than low competitive group.

High competitive group appraised their cognitive anxiety symptoms and their self-confidence as more facilitating than low competitive group.

47 Differences according to playing positions were found concerning vigor (higher for defensive players than offensive ones) and anger (higher for linebackers and offensive backs than for quarterbacks and defensive backs).

48 Self-confidence significantly increased and performance significantly decreased during the tournament.

Somatic and cognitive anxiety decreased during the tournament.

Somatic anxiety was inversely correlated (-.49) to performance when players played under their capacities. 
stressors were identified within the six higher order-themes of health, performance/ outcomes, individual errors, interpersonal relationships, others' behaviors and noncompetitive antecedents (Table 3). The following sections describe the main findings associated with each theme.

Health. Investigations regarding emotional antecedents reported that health was a source of strain for players. Specifically, experiencing pain, sustaining an injury, or worrying about getting an injury were identified as three of the five most frequently reported antecedents/stressors (Noblet $\&$ Gifford, 2002). For example, Anshel (2001) found that experiencing pain comprised $14 \%$ of all stressors reported and was the fourth most common stressor. Similar results were also found for sustaining an injury, which comprised $9.80 \%$, and $13.04 \%$ of all match stressors (and $18.07 \%$ of all training stressors) reported in Nicholls and Polman (2007b) and Nicholls, Jones, Polman and Borkoles (2009), respectively. Moreover, this stressor was ranked as the fourth most common competitive stressor (the third most common training stressor). Finally, Nicholls, Holt, Polman and Bloomfield (2006) found that players considered injuries to be the most important stressor (17.90\%). Similarly, the probability of being injured is also a worry in ice hockey and Canadian football (Dunn, 1999; Dunn \& Syrotuik, 2003)

Performance/Outcome. In line with the CMRT, players reported that the importance of the situation is an important emotional antecedent. For example, Jackson (2003) noted that the degree of importance assigned on a rugby union

\section{Table 3 Emotional Antecedents in Contact Team-Based Sports}

\begin{tabular}{|c|c|c|c|}
\hline $\begin{array}{l}\text { Higher order } \\
\text { themes }\end{array}$ & Antecedents & Studies & $\begin{array}{c}\text { \% of reviewed } \\
\text { investigations } \\
\text { on emotional } \\
\text { antecedents }(n=17)\end{array}$ \\
\hline Health & Health, injury, pain. & $\begin{array}{l}8,9,14,20 \\
24,28,30,32 \\
35\end{array}$ & $52.9 \%$ \\
\hline $\begin{array}{l}\text { Performance/ } \\
\text { outcome }\end{array}$ & $\begin{array}{l}\text { Selection, outcomes, determinant } \\
\text { skills. }\end{array}$ & $\begin{array}{l}2,22,27,30 \\
37,42\end{array}$ & $35.3 \%$ \\
\hline Individual errors & Physical errors, mental errors. & $\begin{array}{l}8,14,20,22 \\
24,28,31,32 \\
35\end{array}$ & $52.9 \%$ \\
\hline $\begin{array}{l}\text { Influence of } \\
\text { others }\end{array}$ & $\begin{array}{l}\text { Negative aspects of relation- } \\
\text { ships, conflicts, criticisms from } \\
\text { others, referee's wrong decisions, } \\
\text { opponents' cheating, opponents' } \\
\text { performance, teammates' behav- } \\
\text { ioral events. }\end{array}$ & $\begin{array}{l}4,8,9,14,18 \\
24,28,30,32 \\
35\end{array}$ & $58.8 \%$ \\
\hline $\begin{array}{l}\text { Noncompetitive } \\
\text { antecedents }\end{array}$ & $\begin{array}{l}\text { Diet, weather, sleep, organiza- } \\
\text { tion, career, work and work/non- } \\
\text { work interface. }\end{array}$ & $8,9,30$ & $17.6 \%$ \\
\hline
\end{tabular}


place-kick influenced players' kicking routines. Valance, Dunn and Causgrove Dunn (2006) also showed that anger is more intense after a personal mistake in a critical situation compared with one in an unimportant situation. Other studies have shown that the outcome of the match is at the origin of the players' emotional experiences (Kerr \& Schaik, 1995; Wilson \& Kerr, 1999). Players report different emotional valences according to the result of the match (i.e., positive when they win and negative when theylose; Wilson \& Kerr, 1999). Gaudreau, Amiot and Vallerand (2009) have also shown that being, or not being selected for a representative team influence players' affective experiences.

Individual Errors. Several studies have shown that players frequently express fear of making a physical error (Lonsdale \& Howe, 2004). Indeed, fear of making an error has been shown to be the most frequent stressor experienced, accounting for $28.04 \%$ and $32 \%$ of all stressors in Nicholls and Polman (2007b) and Anshel's (2001) studies respectively. In addition, mental errors accounted for $14.36 \%$ and $11.89 \%$ of all stressors and represented the second and third most common stressors in studies by Nicholls et al. (2006) and Nicholls and Polman (2007b), respectively. Dunn and Syrotuik (2003) and Dunn (1999) have also reported that fear of failure influences player anxiety, as mistakes were perceived to be associated with a decrease in collective team performance.

The Influence of Others. Several studies have highlighted the infl of teammates on individual player emotions. For example, the quality of team members' relationships and their conflicts have been identified to influence player anxiety (Lonsdale \& Howe, 2004; Noblet \& Gifford, 2002). Specifically, negative social evaluations influence a player's emotional state (Dunn, 1999; Dunn \& Syrotuik, 2003). For example, being criticized by teammates or coaches affects players' emotional states (Dunn, Gotwals, Causgrove Dunn, \& Syrotuik, 2006; Nicholls, Backhouse, Polman, \& McKenna, 2009; Nicholls, Jones et al., 2009; Nicholls \& Polman, 2007b; Noblet \& Gifford, 2002). Interestingly, however, Anshel (2001) noted that reprimands from a coach were not a frequent stressor for adult performers.

A final theme identified under the category of the influence of others was the effect of uncontrollable events/occurrences caused by teammates, opponents, referees, or supporters behavior upon player emotions. Interestingly, players report opponent performance or cheating as a frequent stressor, accounting for $33.00 \%$, $10.73 \%$, and $11.59 \%$ ofall stressors in Anshel (2001), Nicholls, Jones etal. (2009), and Nicholls and Polman (2007b), respectively.

Nonsport Antecedents. Nonsport concerns reported in the literature include major life stressors such as diet, weather and sleep (Nicholls \& Backhouse et al., 2009). Nicholls, Jones et al. (2009) reported weather was also an emotional antecedent in team contact sports. These results complement earlier research showing that the organization, culture, career, development concerns, or the professional nature of the practice, as well as player relationships with supporters, media and sponsors were major nonsport stressors (Noblet \& Gifford, 2002). 


\section{Moderators of Competition-Related Emotional States}

The general literature on CMRT highlights the potential moderating influence of numerous individual and situational variables on performers' primary and secondary appraisals (Lazarus, 1999). In the current review, we identified players' perfectionism trait and player age as the main individual moderators of emotions. In addition, the literature highlights four situational moderators: game location, level of competition, group processes, and the temporal nature of emotional processing.

Individual Characteristics. In line with Lazarus's (1999) suggestions that a person's characteristics influence the appraisal process and their subsequent emotional experience, we identified that players' perfectionism and age influence their emotions in team contact sports.

Specifically, high perfectionist tendencies affect experiences of anger, particularly frustrating and negatively evaluated situations (Valance et al., 2006). Dunn et al. (2006) also showed that perfectionism was correlated with anger such that perfectionists believed that errors should not happen. Moreover, because they feel impotent if not at their best, perfectionists reported experiencing more anger after a mistake in a critical situation (Valance et al., 2006). In addition to anger-related appraisals, player traits have also been reported to influence aggressive behaviors (Maxwell\&Visek, 2009). For example, Bushman \&Wells (1998) reported that trait aggressiveness predicted aggressive penalty minutes in ice hockey.

In relation to player age also influencing emotional experiences, young players have been reported to experience higher anxiety states, be more sensitive to other stressors, and have fewer coping skills than older players (Géczi, Bognár, Tóth, Sipos, \& Fügedi, 2008). Géczi et al. (2008) showed that levels of anxiety and anger differed between youth and adult players during an ice hockey training camp. The authors also found that youth players were more anxious and angry compared with adult players who were better able to cope with their emotions in critical moments. Although these authors found a positive relationship between age and player coping skills as well as a negative relationship between age and anxiety intensity, it is important to note that another study found no relationship between emotions, coping and age (Géczi, Tóth, Sipos, Fügedi, Dancs, \& Bognár, 2009).

Game Location. The influence of game location on the appraisal processes was via players reporting more anxiety in the week leading up to the match when playing away compared with when playing at home (Polman, Nicholls, Cohen, \& Borkoles, 2007). Moreover, players accurately reported an iceberg mood state profi (i.e., high level of vigor combined with low level of the others negative moods; Terry et al., 1998) and elevated aggression at home versus on the road during the precompetitive period (Jones, Bray, \& Olivier, 2005). Finally, playing at home has also been associated with reduced activation states in both pre-and postcompetitive periods compared with away matches (Kerr \& Schaik, 1995).

Level of Competition. The literature also reports that player skill level is a moderating influence on players' appraisal and coping mechanisms. For example, 
some studies found that elite players interpreted their anxiety symptoms as more facilitating than nonelite players (Neil, Mellalieu, \& Hanton, 2006). Golby and Sheard (2003) also found that elite performers perceived match situations as more challenging than threatening, whereas other studies suggest that the players have a greater capacity to manage their emotions effectively (D'Urso, Petrosso, \& Robazza, 2002; Kerr \&Schaik, 1995). Moreover, elite players tend to cope with more cognitive-restructuring-based strategies (Mellalieu, Hanton, \& Thomas, 2009; Neil et al., 2006), whereas nonelite players tend to cope using physical relaxationbased tools (Neil et al., 2006).

Group Processes. At present, the influence that the psychosocial variables connected with interpersonal situations have on emotions has received little attention (Mellalieu, Hanton, \&Shearer, 2008; Vallerand\& Blanchard, 1999). Todate, only collective efficacy and group cohesion have been considered in relation to individual emotional experiences. For example, collective efficacy has been found to explain $22 \%$ of the variance of players' positive affective states experienced (Greenlees, Nunn, Graydon, \& Maynard, 1999).

Interpretation of anxiety symptoms has been reported to influenced players' attraction to the group and group integration components of task-cohesion (ATG-T and $\mathrm{GI-T}$ respectively, which refer to the common investment in the attainment of a team goal; Eys, Hardy, Carron, \& Beauchamp, 2003; Terry et al., 2000). Furthermore, perception of group cohesion explained $2.56-12.45 \%$ of the variation in the players' emotional states, with task cohesion correlated with anger $(r=-.19$; Terry et al., 2000).

The temporal Nature of Emotions. Some of the studies that we reviewed (e.g., Nicholls, Backhouse et al., 2009; Nicholls, Jones et al., 2009) showed that the experience of various stressors differed between rest, training and competitive periods. For example, Nicholls, Jones et al. (2009) found that anxiety symptoms differed between training and competitive periods. They also found that coping was more effective during training sessions, emotions were more intense during matches, and emotions were more frequent on training days (Table 4).

\section{Emotions Experienced By Players}

The current results showed that $62,50 \%$ of the studies we reviewed analyzed the emotions experienced by players (e.g., Géczi et al., 2008; Mellalieu et al., 2008; Nicholls, Jones et al., 2009; Robazza, Bertollo, \& Bortoli, 2006; Valance et al., 2006). Although some of the studies examined experiences of emotion-related constructs such as mood or positive/negative affect (e.g., Polman et al., 2007; Gaudreau et al., 2009), only two emotions consistent with Lazarus's CMRT (1999) were reported: anger (25.00\%) and anxiety (42.50\%).

Anger and Anxiety. Players frequently report feeling angry (Grange \& Kerr, 2010; Robazza et al., 2006; Robazza \& Bortoli, 2007) or anxious (e.g., Anshel, 2001; Mellalieu et al., 2008; Nicholls, Backhouse et al., 2009). These emotions were sometimes associated with the study of the direct perception of symptom intensity, which could either be interpreted as debilitative or facilitative. More specifically, players considered the somatic anxiety and anger experienced in precompetitive 
Table 4 Differences between Training and Match Periods Relating to Emotions and Coping in Contact Team-Based Sports

\begin{tabular}{lcc}
\hline & Training & Match \\
\hline Emotion intensity & - & + \\
Emotion frequency & + & - \\
Coping effectiveness & + & - \\
\hline
\end{tabular}

periods as facilitative (Eys et al., 2003; Hanton, Jones, \& Mullen, 2000; Jones \& Swain, 1992) because of the high levels activation required for the contact nature of rugby union, and the feelings associated with these affective states (Neil et al., 2006; Maxwell \& Visek, 2009; Robazza \& Bortoli, 2007). Wealso found that players often felt angry during matches (Bushman \& Wells, 1998; Valance et al., 2006).

Other Emotions. Our review did not fi studies addressing other CMRTacknowledged emotions in team contact sports. However, several studies report that players experience both positive and negative affects (Gaudreau et al., 2009; Hagtvet \& Hanin, 2007; Hanin \& Syrjä, 1995; Nicholls, Jones et al., 2009) and mood states (Polman et al., 2007; Terry et al., 2000; Terry, Walrond, \& Carron, 1998). Players' emotional states other than CMRT-associated emotions, such as tension, sullenness or relaxation, seemed to change over time (e.g., D'Urso et al., 2002; Kerr \& Svebak, 1994; Maynard \& Howe, 1987; Wilson \& Kerr, 1999). For example, tension tends to decrease from pre- to postcompetitive periods (Wilson \& Kerr, 1999).

\section{Coping Responses}

We reviewed ten papers that focused on players' coping responses in team contact sports. The results suggest that coping responses are in accordance with CMRT in so far as appraisal and coping interact during emotional processes (Anshel, 2001). Specifically, players used a range of strategies to cope with their emotions (Table 5): Problem-focused coping (60\%) was more prevalent than emotion-focused (40\%) or avoidance-focused coping (30\%; Nicholls et al., 2006). Interestingly, the perceived effectiveness of the coping strategies employed has been investigated. For example, in their study of rugby players, Nicholls et al. (2006) showed that players did not view their coping strategies as effective. They also indicated that despite using three coping strategies (i.e., blocking, positive reappraisal and increased effort) to deal with three significant stressors (i.e., physical errors, mental errors, and injuries), only increased effort was perceived to be effective. This finding suggests that the professional male rugby union players studied did not know how to cope efficiently with their emotions to produce a successful performance.

\section{Discussion}

The purpose of this study was to systematically review the scientific literature on the emotional process in team contact sports, and to offer recommendations forfuture 
Table 5 Coping in Contact Team-Based Sports

\begin{tabular}{|c|c|c|c|c|}
\hline $\begin{array}{c}\text { Functional } \\
\text { role }\end{array}$ & $\begin{array}{l}\text { Types of } \\
\text { efforts }\end{array}$ & Strategies used & Studies & $\begin{array}{l}\% \text { of reviewed } \\
\text { investigations } \\
\text { on coping } \\
(n=10)\end{array}$ \\
\hline \multirow[t]{2}{*}{$\begin{array}{l}\text { 1. Problem- } \\
\text { focused }\end{array}$} & Behavioral & $\begin{array}{l}\text { Increasing efforts, routines, } \\
\text { taking advice, technique } \\
\text { oriented coping, changing } \\
\text { behaviors, communicating. }\end{array}$ & $\begin{array}{l}8,12,14,20 \\
27\end{array}$ & $50 \%$ \\
\hline & Cognitive & $\begin{array}{l}\text { Increasing attention, focus- } \\
\text { ing on his role, mental } \\
\text { imagery, instructional self- } \\
\text { talk, planning. }\end{array}$ & $8,14,20,32$ & $40 \%$ \\
\hline \multirow{2}{*}{$\begin{array}{l}\text { 2. Emotion- } \\
\text { focused }\end{array}$} & Behavioral & Relaxation. & 19 & $10 \%$ \\
\hline & Cognitive & $\begin{array}{l}\text { Reappraisal, external verbal } \\
\text { persuasion, mental imagery, } \\
\text { self-talk, blaming. }\end{array}$ & $7,11,19,20$ & $40 \%$ \\
\hline \multirow{2}{*}{$\begin{array}{l}\text { 3. Avoid- } \\
\text { ance }\end{array}$} & Behavioral & Withdrawal. & 20 & $10 \%$ \\
\hline & Cognitive & Blocking, thought stopping. & $8,14,20$ & $30 \%$ \\
\hline
\end{tabular}

research and applied interventions. One of the main findings was that anger and anxiety are the predominantly studied emotions in the field of team contact sports. However, it is unclear whether these emotions are the most popular emotions investigated, or whether they are central to player performance experiences (Robazza \& Bortoli, 2007). With regard to the former suggestion, the existing literature would seem to indicate that further exploration regarding experiences of participating in team contact sport is required. Indeed, Lazarus (1999) has suggested that 7 emotions (i.e., anger, anxiety, guilt, shame, relief, happiness, and pride) influence sport performance (Lazarus, 2000). Applying CMRT to team contact sports appears to be a good basis from which the prevalence and influence of other emotions can be investigated. For example, if we consider the aggressive aspects of these sports and their associated risks, exploring the emotion of fright would be relevant (i.e., "an immediate, concrete, and overwhelming physical danger"; Lazarus, 1999, p. 96). Similarly, if we consider the interpersonal dimension of team contact sports, investigating emotions such as guilt or compassion would also be interesting. In relation to the latter suggestion that anger and anxiety are central to performance, this review indicates that elite players appraise both anger and anxiety as facilitating performance, which is coherent with the general sport psychology literature 
(Hanin 2000). Indeed, anxiety is the most studied emotion in sport. After initially being considered as detrimental to performance, studies have indicated that anxiety is frequently associated with successful performance (e.g., Hardy, 1997). Both Hanin (2000) and Jones (1995) have outlined how the intensity of anxiety exerts a strong influence on performance; furthermore, athletes may appraise anxiety symptoms as positive in relationship to an upcoming performance. Lazarus (2000) corroborated these suggestions when he proposed that anxiety and anger influence sports performance by mobilizing energy. With regards to anger, it is frequently experienced in contact sports (Isberg, 2000) and considered to be one of the optimal sport-related emotions according to the Individual Zones of Functioning model (IZOF, Hanin, 2000). However, despite the fact that some players consider anger as facilitating, the increase of sanctioned aggressive acts also indicates that this emotion may lead players to adopt behaviors that damage both individual and team performance. These results are in accordance with the literature on anger in sports, which is often studied via aggressive behavior (e.g., Jones, 2003; Maxwell, 2004). In summary, our review regarding the emotions experienced in team contact sports (specifically, anger and anxiety) suggests three applied practical implications: (1) coaches should induce anger and anxiety during competition; (2) players must be taught to recognize emotional responses and their associated behaviors; and (3) players must be taught to control their aggressiveness when they are too angry (for more detail on anger management, see Isberg, 2000, p. 131-132).

A second finding from our review is that most of the studies in this area investigated the precompetitive period. Because they appear to be markedly different from the emotions experienced during a match (D'Urso et al., 2002), several researchers have questioned the influence that precompetitive emotions have on subsequent performance (e.g., Debois, 2003). Therefore, we encourage future research to consider other periods in addition to the precompetitive phase, and to explore the influence that each period has on the other. For example, a study could investigate postcompetitive emotions versus the precompetitive emotions of the following game. In addition, in line with Nicholls, Backhouse et al.'s (2009) study that focused on extracompetitive periods, we suggest that coaches and applied practitioners take into account players' emotional experiences during both training and rest periods among professionals or elite youth who live at an elite training structure (e.g., union or professional club training centers). Indeed, because of the high stakes associated with competing for a professional career in sport, and because of the pressure to comply with the demands at the elite level, varied and frequent emotional states are reported as being experienced (Creswell \& Eklund, 2006). Studying the emotional dynamics related to these practices can therefore provide important knowledge to optimize player performances. For example, this could help coaches to avoid burnout among their players (Creswell \& Eklund, 2006), or better control the emotional impact of the pressure associated with the training environment on the attainment of players' optimal competitive emotional states (Hanin, 2000).

A third finding of our review suggests that, due to the difficulties associated with measuring coping effectiveness (Nicholls et al., 2006; Nicholls \& Polman, $2007 \mathrm{~b}$ ), we cannot ascertain the influence of coping on team contact sport performance experiences. The review does suggest however, that there is a difference between coping and coping effectiveness because one strategy might effectively 
manage emotions in one situation, but not with regard to sport performance (Nicholls, Backhouse et al., 2009). Consequently, to help players effectively cope with their emotions, we encourage researchers to explore the relationship between coping strategies and team contact sports performance. In addition, the results from our review concerning player ability to cope with their emotions showed that they used many strategies. Thus, researchers should identify which emotion regulation strategies should be used for specific emotional experiences according to specific game incidents. The results of these studies would allow coaches and applied practitioners to create interventions to enable players to cope effectively with their emotions. For example, if a player makes a mistake in a game (e.g., a missed tackle), and subsequently attempts to make up for this by initiating individual actions that deviate from the team strategy, the coach may wish to focus on reappraising the original error made by down playing the importance of the mistake. Although several researchers have studied interpersonal emotion processes within sports teams and groups in general (e.g., interpersonal and intragroup anxiety; Hanin, 1989; emotional intelligence in sports teams; Latimer, Rench, \& Brackett, 2007; and emotional contagion; Totterdell, 2000), more research is needed to summarize the group processes that affect players' emotions (Mellalieu et al., 2008). Lazarus (2000) not only used CMRT to highlight the importance of the situation, but also proposed that the attribution of accountability influences a person's emotional experience. The degree of personal responsibility is particularly evident in the distinction between individual and team sports: A player's control over the performance of the team as a whole varies throughout the match. Others may also influence emotion regulation (Gross \& Thompson, 2007). For example, a player might cope by managing his or her emotions alone, by sharing them (Rimé, 2009) or by being helped by a teammate to cope (Niven et al., 2009). Although researchers have already recognized the importance of the group to performance, we suggest that future research focus on group-level emotional experience in team contact sports (Smith, Seger, \& Mackie, 2007). Researchers should also explore the influence that others have on player emotion and emotion regulation (Barsade \& Gibson, 1998). From an applied perspective, given a group's impact on emotional processes, our findings suggest that a focus on promoting the components of task cohesion (i.e., ATC-t and Gl-t) will facilitate positive interpretations of precompetitive emotions experienced. This focus can also be applied to intergroup emotion and intergroup emotion regulation. For example, are a player's emotions related to their social identification as a member of country's team or merely as a player? (Smith et al., 2007). Similarly, does a player regulate his or her emotions according to team norms through group identification and the development of social norms? (Stephens \& Light-Bredemeier, 1996).

Finally, our review highlights another potential moderator of the emotional process, the skill level of the specific sport being studied (Cerin et al., 2000). Many of the papers that we reviewed focused on adult populations. And while some discussed young adults and adolescent athletes, no study examined children. Only a few studies investigated skill-level differences in emotion, especially comparing youth and adult players (Géczi et al., 2008; Géczi et al., 2009). Frequently, children have not yet acquired the necessary coping skills, and it seems plausible that the collision events, on which contact team sports are based, may significantly affect the emotional states of a child who has not yet learnt to regulate his emotions (Goyen \&Anshel, 1998; Nicholls \& Polman, 2007a). 


\section{Limitations}

Although we adopted a broad field of search in our review via $11 \times 6$ search terms (i.e., emotion terms $x$ specific-sport terms) to offer a comprehensive picture of the emotional process in contact team sports, we recognize that a range of variables affect the emotional process (Lazarus, 1999), which we were unable to fully cover in the present paper. Future studies may therefore wish to consider searching for additional variables to further refine understanding of the emotional experience associated with team sport performance, such as the "home advantage" or "perfectionism".

\section{Conclusion}

The study of emotional experiences in sports has received considerable attention over the past 25 years. However, because of differences across specific sports, findings have been inconsistent from one sport to another. Consequently, our review aimed to account for particular sports characteristics to provide recommendations for future research and interventions in the field of emotions in sport. To this extent, we outlined a classification system that allows researchers to collect and synthesize results from similar, albeit unique, sports (in this case, team contact sports) and consider each result as indicative of this type of sport. We also attempted to provide a starting point from which to develop a comprehensive knowledge base regarding the emotional processes involved in team contact sports performance. Furthermore, we recommend several areas for additional research including: (1) studying different emotions; (2) investigating different time periods; (3) exploring emotion-specific regulation strategies; (4) considering level of playing experience; and (5) focusing on the influence of others on player's emotion and emotion regulation. Finally, we identified four intervention possibilities within team contact sports: (1) induce anger and anxiety to positively influence performance; (2) teach players how to recognize and regulate their emotions, especially anger and anxiety; (3) focus on extracompetitive stressors; and (4) monitor the effectiveness of existing player coping strategies and developemotion-specific regulation strategies.

\section{References}

References marked with an asterisk indicate studies included in the review.

*. Anshel, M.H. (2001). Qualitative validation of a model for coping with acute stress in sport. Journal of Sport Behavior, 24, 223-246.

Barsade, S.G., \& Gibson, D.E. (1998). Group emotion: A view from top and bottom. In D.H. Gruenfeld, M. Neale, \& E. Mannix (Eds.), Researchon managing groupsandteams (Vol. 1, pp. 81-102). Greenwich, CT:JAI Press.

Bland, C.J., Meurer, L.N., \& Maldonado, G. (1995). A systematic approach to conducting a non-statistical meta-analysis of research literature. Academic Medicine, 70, 642-653.

Biddle, S. (2000). Exercise, emotions, and mental health. In Y.L. Hanin (Ed.), Emotions in sport (pp. 39-64). Champaign, IL: Human Kinetics.

*. Bushman, B.J., \&Wells, B.L.(1998). Traitaggressiveness and hockeypenalties: Predicting hot tempers on the ice. The Journal of Applied Psychology, 83, 969-974. 
Cerin, E., Szabo, A., Hunt, N., \& Williams, C. (2000). Temporal patterning of competitive emotions: A critical review. Journal of Sports Sciences, 18, 605-625.

Conquet, P., \& Devaluez, J. (1978). Les fondamentaux du rugby. Paris: Vigot. [The basics of rugby union].

Cresswell, S.L., \& Eklund, R.C. (2006). The nature of player burnout in rugby: Key characteristics and attributions. Journal of Applied Sport Psychology, 18, 21 9-239.

Debois, N. (2003). De l'anxiété aux émotions compétitives: Etat de la recherche sur les états affectifs en psychologie du sport [From anxiety to competitive emotions: Sport psychology research about affects]. STAPS, 62, 21-41.

*. D'Urso, V., Petrosso, A., \& Robazza, C. (2002). Emotions, perceived qualities and performance of rugby players. The Sport Psychologist, 16, 173-1 99.

*. Dunn, J.G.H. (1999). A theoretical framework for structuring the content of competitive worry in ice hockey. Journal of Sport \& Exercise Psychology, 21, 259-279.

*. Dunn, J.G.H., \& Nielsen, A.B. (1993). A between-sport comparison of situational threat perceptions in ice hockey and soccer. Journal of Sport \& Exercise Psychology, 15, 449-465.

*. Dunn, J.G.H., Gotwals, J.K., Causgrove Dunn, J., \& Syrotuik, D.G. (2006). Examining the relationship between perfectionism and trait anger in competitive sport. International Journal of Sport and Exercise Psychology, 4, 7-24 .

Dunn, J.G.H., \& Nielsen, A.B. (1996). A classificatory system of anxiety-inducing situations in four team sports. Journal of Sport Behavior, 19, 11-131.

*. Dunn, J.G.H., \& Syrotuik, D.G. (2003). An investigation of multidimensional worry dispositions in a high contact sport. Psychology of Sport and Exercise, 4, 265-282.

*. Eys, M.A., Hardy,J., Carron, A.V., \& Beauchamp, M.R. (2003). The relationship between task cohesion and competitive state anxiety. Journal of Sport \& Exercise Psychology, 25, 66-76.

Eysenck, H.J. (1995). Problems with meta-analysis. In I. Chalmers \& G.G. Altman (Eds.), systematic reviews (pp. 64-74). London: BMJ Publishing Group.

Famose, J.P. (1990). Apprentissage moteur et difficulté de la tâche. Paris: INSEP. [Motricity learning and the task difficulty].

*. Freudenberger, L.F., \& Bergandi, T.T. (1994). Sport psychology research in American football:A review of the literature. International Journal of Sport Psychology, 25, 425-434.

Frijda, N.H. (1994). Varieties of affect: Emotions and episodes. Moods and sentiments. In P. Ekman \& R.J. Davidson(Eds.), The nature of emotion (pp. 59-67). Oxford, England: Oxford University Press.

*. Gaudreau, P., Amiot, A.E., \& Vallerand, R.J. (2009). Trajectories of affective states in adolescent hockey players: Turning point and motivational antecedent. Developmental Psychology, 45, 307-319.

*. Géczi, G., Bognár, J., Tóth, L., Sipos, K., \& Fügedi, B. (2008). Anxiety and coping of Hungarian national ice hockey players. International Journal of Sports Science \& Coaching, 3, 277-285.

*. Géczi, G., Tóth, L., Sipos, K., Fügedi, B., Dancs, H., \& Bognár,J. (2009). Psychological profiles of Hungarian national young ice hockey players. Kinesiology, 41, 87-94.

*. Golby, J., \& Sheard, M. (2003). Mental toughness and hardiness at different levels of rugby league. Personality and Individual Differences, 37, 933-942.

*. Golby, J., Sheard, M., \& Lavallee, D. (2003). A cognitive-behavioral analysis of mental toughness in national rugby league football teams. Perceptual and Motor Skills, 96, 455-462.

Goyen, M.J., \& Anshel, M.H. (1998). Sources of acute competitive stress and use of coping strategies as a function of age and gender. Journal of Applied DevelopmentalPsychology, 19, 469-486.

*. Grange, P., \& Kerr,J.H. (2010). Physical aggression in Australian football: A qualitative study of elite athletes. Psychology of Sport and Exercise, 11, 36-43. 
*. Greenlees, I.A., Nunn, R.L., Graydon, J.K., \& Maynard, I.A. (1999). The relationship between collective efficacy and precompetitive affect in rugby players: Testing Bandura's model of collective efficacy. PerceptualandMotor Skills, 89, 431-440.

Gross, J.J., \& Thompson, R.A. (2007). Conceptual foundations. In J.J. Gross (Ed.), Handbook of emotion regulation (pp. 3-26). NewYork, NY: The Guilford Press.

*. Hagtvet, K.A., \& Hanin, Y.L. (2007). Consistency of performance-related emotions in elite athletes: Generalizability theory applied to IZOF model. Psychology of Sportand Exercise, 8, 47-72.

Hanin, Y.L. (1989). Interpersonal and intragroup anxiety in sports. In D. Hackfort \& C.D. Spielberger (Eds.), Anxiety in sports: an international perspective (pp. 19-28). New York, US: Hemisphere Publishing Corporation.

Hanin, Y.L. (2000). Emotions in sport. Champaign, IL: Human Kinetics.

*. Hanin, Y., \& Syrjä, P. (1995). Performance affect in junior ice-hockey players: An application of the Individual Zones of Optimal Functioning Model. The Sport Psychologist, 9, 169-187.

*. Hanton, S., Jones, G., \& Mullen, R. (2000). Intensity and direction of competitive anxiety as interpreted by rugby players and rifle shooters. Perceptual and Motor Skills, 90 , 513-521.

Hardy, L. (1997). The Coleman Roberts Griffi address: Three myths in applied consultancy work. Journal of Applied Sport Psychology, 9, 277-294.

Isberg, L. (2000). Anger, aggressive behavior, and athletic performance. In Y.L. Hanin (Ed.), Emotions in sport (pp. 113-134). Champaign, IL: Human Kinetics.

*. Jackson, R.C. (2003). Pre-performance routine consistency: Temporal analysis of goal kicking in the rugby union World Cup. Journal of Sports Sciences, 21, 805-814.

*. Jones, G., \& Swain, A. (1992). Intensity and direction as dimensions of competitive anxiety and relationships with competitiveness. Perceptual and Motor Skills, 74, 467-472.

Jones, M.V. (1995). Competitive anxiety in sport. In S.J.H. Biddle (Ed.), European perspectives on exercise and sport psychology (pp. 128-153). Leeds, England: Human Kinetics.

Jones, M.V. (2003). Controlling emotions in sport. The Sport Psychologist, 17, 471-486.

*.Jones, M.V., Bray, S.R., \& Olivier, S. (2005). Game location and aggression in rugby league. Journal of Sports Sciences, 23, 387-393.

*. Kerr, J.H., \& Schaik, P. (1995). Effects of game venue and outcome on psychological mood states in rugby. Personality and Individual Differences, 19, 407-410.

*. Kerr,J.H., \& Svebak, S. (1994). The acute effects of participation in sport on mood: The importance of level of antagonistic physical interaction. Personality and Individual Differences, 16, 159-166.

Krauss, M.D. (2004). Equipment innovations and rules changes in? sports. Current Sports Medicine Reports, 3, 272-276.

Lane, A.M., Beedie, C.J., \& Stevens, M.L. (2005). Mood matters: A response to Mellalieu. Journal of Applied Sport Psychology, 17, 319-325.

*. Lane, A.M., Rodger,J.S.E., \& Karageorghis, C.I. (1997). Antecedents of state anxiety in rugby. Perceptual and Motor Skills, 84, 427-433.

Latimer, A., Rench, T., \& Brackett, M.A. (2007). Emotional intelligence-A framework for examining emotions in sport and exercise groups. In M. Beauchamp \& M. Eys (Eds.), Group dynamics in exercise psychology: Contemporary themes (pp. 3-24). London, UK: Routledge.

*. Lauer, L., \& Paiement, C. (2009). The playing tough and clean hockey program. The Sport Psychologist, 23, 543-561.

Lazarus, R.S. (1991). Emotion and adaptation. NewYork, NY: Oxford UniversityPress.

Lazarus, R.S. (1999). Stress and emotion: A new synthesis. New York, NY: Springer Publishing Co.

Lazarus, R.S. (2000). How emotions influence performance in competitive sports. The Sport Psychologist, 14, 229-252. 
Lazarus, R.S., \& Folkman, S. (1984). Stress, appraisaland coping. NewYork, NY: Springer.

*. Lonsdale, C., \& Howe, B.L. (2004). Stress and challenge appraisals of acute taxing events in rugby. International Journal of Sport and Exercise Psychology, 2, 7-23.

Mann, D.T.Y., Williams, A.M., Ward, P., \&Janelle, C.M. (2007). Perceptual-cognitive expertise in sport: A meta-analysis. Journal of Sport \& Exercise Psychology, 29, 457-478.

Martens, R., Vealey, R.S., \& Burton, D. (1990). Competitive anxiety in sport. Champaign, IL: Human Kinetics.

Maxwell,J.P.(2004). Anger rumination. Psychology of Sport and Exercise, 5, 279-289.

*. Maxwell, J.P., \& Visek, A.J. (2009). Unsanctioned aggression in rugby union: Relationships among aggressiveness, anger, athletic identity, and professionalization. Aggressive Behavior, 35, 237-243.

*. Maxwell, J.P., Visek, A.J., \& Moores, E. (2009). Anger and perceived legitimacy of aggression in male Hong Kong Chinese athletes: Effect of type of sport and level of competition. Psychology of Sport and Exercise, 10, 289-296.

*. Maynard, I.W., \& Howe, B.L. (1987). Interrelations of trait and state anxiety with game performance of rugby players. Perceptual and Motor Skills, 64, 599-602.

*. McGowan, R.W., \& Shultz, B.B. (1989). Task complexity and affect in collegiate football. Perceptual and Motor Skills, 69, 671-674.

*. Mellalieu, S.D., Hanton, S., \& Shearer, D.A. (2008). Hearts in fire, heads in the fridge. A qualitative investigation into the temporal patterning of the precompetitive response in elite performers. Journal of Sports Sciences, 26, 81 1-824.

*. Mellalieu, S.D., Hanton, S., \& Thomas, O. (2009). The effects of a motivational generalarousal imagery intervention upon preperformance symptoms in male rugby union players. Psychology of Sport and Exercise, 10, 1-11.

Mulrow, C.D. (1995). Rationale for systematic reviews. In I. Chalmers \& D.G. Altman (Eds.), Systematic reviews (pp. 1-8). London: BMJPublishing Group.

*. Neil, R., Mellalieu, S.D., \& Hanton, S. (2006). Psychological skills usage and the competitive anxiety response as a function of skill level in rugby union. Journal of Sports, Science, and Medicine, 5, 415-423.

*. Nicholls, A.R., Backhouse, S.H., Polman, R.C.J., \& McKenna, J. (2009). Stressors and affective states among professional rugby union players. Scandinavian Journal of Medicine \& Science in Sports, 19, 121-128.

*. Nicholls, A.R., Holt, N.L., Polman, R.C.J., \& Bloomfield, J. (2006). Stressors, coping, and coping effectiveness among professional rugby union players. TheSportPsychologist, 20, 314-329.

*. Nicholls, A.R., Jones, C.R., Polman, R.C.J., \& Borkoles, E. (2009). Acute sport-related stressors, coping, and emotion among professional rugby players during training and matches. Scandinavian JournalofMedicine \& Science in Sports, 19, 1 13-120.

Nicholls, A.R., \& Polman, R.C.J. (2007a). Coping in sport: a systematic review. Journal of Sports Sciences, 25, 11-31.

*. Nicholls, A.R., \& Polman, R.C.J. (2007b). Stressors, coping and coping effectiveness among rugby players from the England under-18 rugby union team. Journal of Sport Behavior, 30, 199-217.

Niven, K., Totterdell, P., \& Holman, D. (2009). Aclassification of controlled interpersonal affect regulation strategies. Emotion (Washington, D.C.), 9, 498-509 .

*. Noblet, A.J., \& Gifford, S.M.(2002). The sources of stress experienced by professional Australian footballers. Journal of Applied Sport Psychology, 14, 1-13.

Oatley, K., \& Jenkins, J. (1996). Understanding emotions (pp. 108-109). Cambridge, Mass: Blackwell Publishers, Inc.

Oxendine, J.B. (1970). Emotional arousal and motor performance. Quest, 13, 23-32.

Parkinson, B., Totterdell, P., Briner, R.B., \& Reynolds, S. (1996). Changing moods: The psychology of mood and mood regulation. London: Longman. 
*. Polman, R., Nicholls, A.R., Cohen, J., \& Borkoles, E. (2007). The influence of game location and outcome on behavior and mood states among rugby league players. Journal of Sports Sciences, 25, 1491-1500.

Prapavessis, H., \& Grove, J.R. (1994). Personality variables as antecedents of precompetitive mood state temporal patterning. International Journal of Sport Psychology, 25, 347-365.

Rimé, B. (2009). Emotion elicits the social sharing of emotion: Theory and empirical review. Emotion Review, 1, 60-85.

*. Robazza, C., \& Bortoli, L. (2007). Perceived impact of anger and anxiety on sporting performance in rugby players. Psychology of Sport and Exercise, 8, 875-896.

*. Robazza, C., Bertollo, M., \& Bortoli, L. (2006). Frequency and direction of competitive anger in contact sports. Journal of Sports, Medicine, and Physical Fitness, 46, 501-508.

Ruiz, M.C., \& Hanin, Y.L. (2004). Metaphoric description and individualized emotion profiling of performance related states in high-level karate athletes. Journal of Applied Sport Psychology, 16, 1-16.

Smith, E.R., Seger, C.R., \& Mackie, D.M. (2007). Can emotions be truly group level? Evidence regarding four conceptual criteria. Journal of Personality and Social Psychology, 3, 431-446.

Stephens, D.E., \& Light-Bredmeier, B.J. (1996). Moral atmosphere and judgments about aggression in girls' soccer: Relationships among moral and motivational variables. Journal of Sport \& Exercise Psychology, 18, 158-173.

Terry, P.C. (1995). The efficacy of mood state profiling among elite competitors: A review and synthesis. The Sport Psychologist, 9, 309-324.

*. Terry, P.C., Carron, A.V., Pink, M.J., Lane, A.M., Jones, G.J., \& Hall, M.P. (2000). Perceptions of group cohesion and mood in sport teams. Group Dynamics, 4, 244-253.

*. Terry, P.C., Walrond, N., \& Carron, A.V. (1998). The infl of game location on athletes' psychological states. Journal of Science and Medicine in Sport, 1, 29-37.

Totterdell, P. (2000). Catching moods and hitting runs: Mood linkage and subjective performance in professional sport teams. The Journal of Applied Psychology, 85, 848-859 .

*. Uphill, M.A., \& Jones, M.V.(2007). Antecedents of emotions in elite athletes: A cognitive motivational relational theory perspective. Research Quarterly for Exercise and Sport, 78, 79-89.

*. Valance, J.K.H., Dunn, J.G.H., \& Causgrove Dunn, J.L. (2006). Perfectionism, anger and situation criticality in competitive youth ice hockey. Journal of Sport \& Exercise Psychology, 28, 383-406.

Vallerand, R.J., \& Blanchard, C.M. (2000). The study of emotion in sport and exercise: historical, definitional, and conceptual perspectives. In Y.L. Hanin (Ed.), Emotions in sport (pp. 3-38). Champaign, IL: Human Kinetics.

*. Verger, M., \& Bertrank, S. (2008). L'apprentissage d'une routine de performance en rugby à partir des croyances (exemple du buteur). [The training of a routine of performance in rugby starting from the beliefs (example of the striker).]. Science \& Sports, 23, 38-40.

Vom Hofe,A. (1982). Sport et personnalité: étude de la correspondance à partir d'un modèle de classification. [Sport and personality: study of the correspondence from a classification model.]. Sciences et Techniques des Activités Physiques et Sportives, 60, 18-35.

Weed, D.L. (1997). Methodological guidelines for review papers. Journal of the National Cancer Institute, 89, Retrieved from http://svn.cancerprev.org/ www.cancerprev.org/ trunk/pub/Journal/Guidelines/pdf/Weed.pdf.

*. Wilson, G.V., \& Kerr, J.H. (1999). Affective response to success and failure: A study of winning and losing in competitive rugby. Personality and Individual Differences, 27, 85-99. 